\title{
Behind the 3D Scene: A GIS Approach for Managing the Chronological Information of Historic Buildings
}

\author{
Gamze Saygi ${ }^{1,2, *(\mathbb{D})}$, Giorgio Agugiaro ${ }^{3}$ (1) and Mine Hamamcioglu-Turan ${ }^{4}$ \\ 1 Department of Architecture, Faculty of Architecture, Dokuz Eylül University (DEU), 35160 İzmir, Turkey \\ 2 Modèles et simulations pour l'Architecture et le Patrimoine (UMR 3495 CNRS/MCC MAP), \\ The National Center for Scientific Research (CNRS), 13402 Marseille CEDEX 20, France \\ 3 Center for Energy, Austrian Institute of Technology (AIT), 1210 Wien, Austria; giorgio.agugiaro@ait.ac.at \\ 4 Department of Architectural Restoration, Izmir Institute of Technology (IYTE), 35430 Izmir, Turkey; \\ mineturan@iyte.edu.tr \\ * Correspondence: gamze.saygi@map.cnrs.fr; Tel.: +33-0-491-16-43-42
}

Received: 12 April 2018; Accepted: 8 May 2018; Published: 11 May 2018

\begin{abstract}
Historic buildings are representations of cultural systems throughout time. Thus, it is important to shed light on any given historic building's chronological information by means of restitution, that is, the descriptions of different (re)compositions of a building for different historical states. However, the representation of information beyond the geometric, spatial, and physical characteristics of a historic building carries a crucial importance in the holistic understanding of historical changes. This requirement consists of representation, archival, assessment, and management aspects. Nevertheless, covering all these aspects is still quite challenging. Thus, three-dimensional (3D) visualization in digital platforms is considered, which indeed seems to be the most desirable way today. This article proposes a methodology through adopting an approach based on the Geographical Information System (GIS) and further develops a digital "container" as a 3D digital model for the archiving of a building's chronological information. This does not only cover the geometric and spatial aspects, but also the historical resources and their reliability. The paper concludes that the developed prototype may lessen the future investigation effort of heritage specialists by making the chronological information of a historical building more integrated and coherent, through increased readability, accessibility, and visibility. This, in turn, supports the planning of restoration projects, as well as provides a static and comprehensive archive.
\end{abstract}

Keywords: spatio-temporal model; restitution; historic building; transformation; historic changes; building stratigraphy; semantic enrichment; 3D reconstruction; 3D archive

\section{Introduction}

Most heritage buildings incorporate various visible and invisible informative layers from different periods as traces of history. So, what we "see" today is not necessarily "old" architectural entities, but multi-layered representations of the past. Besides, any of the buildings might undergo continuous changes over its lifespan due to adaptations, repair, or conservations. This, in turn, requires a cultural heritage $(\mathrm{CH})$ specialist (for example, an architectural historian or a conservation-architect) to start a journey of research and investigation, to discover, understand, and represent, then to manage and conserve these heritage buildings. A typical investigation covers different, yet complementary, research steps, in which historical information carries a priori importance in supporting both the analysis for spatial and element changes (transformations, removals, additions) in the building's architecture, and the synthesis for (re)establishing the architecture in different historical periods. Briefly stated, the readable and visible historical information and their associated sources related to the physical artifact is of great importance for 
an efficient and complete understanding of the architectural history of any heritage building. A typical investigation (Figure 1) covers different, yet complementary, research steps:

(i) On-site data acquisition,

(ii) Hands-on investigations of the building's characteristics, which result in the production of measured drawings which show the as-found state of the heritage building,

(iii) Complementary archival and historical research, and comparative study for supporting analysis, which is a comprehensive process covering spatial and/or element alterations (transformations, removals, additions), structural failures or deformations, and material deteriorations,

(iv) Synthesis for the (re)establishing of the building's chronology in different historical time intervals, and a critical evaluation of the physical aspects and other aspects beyond those,

(v) Development of intervening decisions, with regard to the proposed conservation actions.

As a consequence, deciphering the evolution of the building chronology is an indispensable part of any conservation activity. Nevertheless, it should not only be considered as an integral activity supporting the intervention decisions of architectural conservation, but also an important form of knowledge and the unique expression of human achievement to transmit to future generations [1]. Correspondingly, the creation of a holistic archive dedicated to understanding the evolution of a historic building has a great importance, and it gives the possibility to use systemized and structured information for an entire perceptive understanding of the different historical states within the building's chronology.

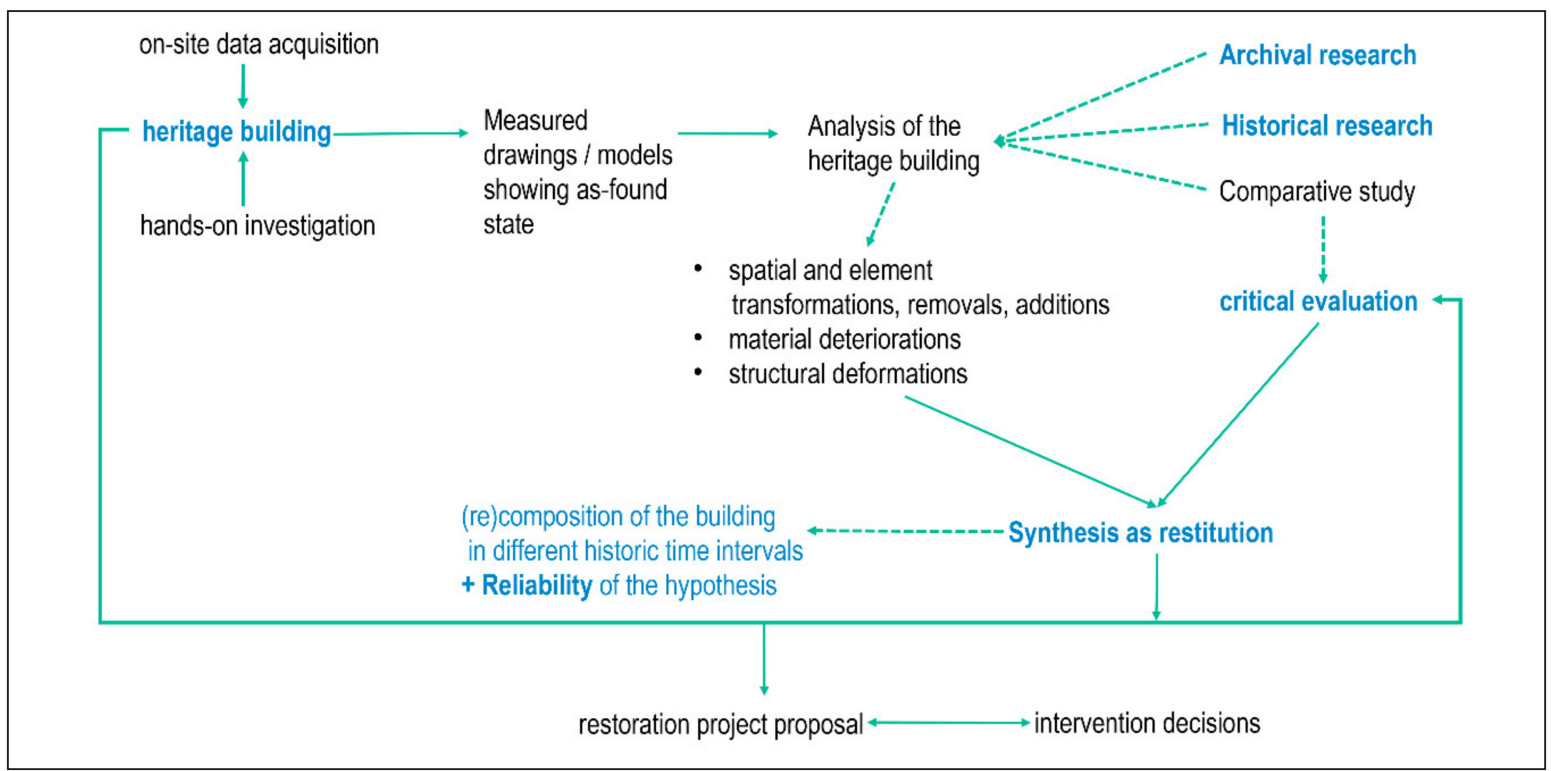

Figure 1. The research process prior to conservation activities.

However, digital reconstructions of heritage buildings in a digital three-dimensional (3D) platform are the most desirable way, as it offers users unrestricted visualization [2]. Yet, pure geometric information is not enough to understand the complex and rich history beyond the physical aspects of the architecture, as it lacks complementary analytical and historical information. The optimal way is thus, to integrate external information sets (for example, textual, graphical, pictorial) by means of a semantically enriched 3D model, which will provide the possibility of comprehensive accessibility for heritage specialists in visualizing, querying, and enhancing, all in line with the final intent being that of a complete understanding. 


\subsection{State-of-the-Art}

As of today, the advances in Information Communication Technologies (ICT) allows heritage researchers to produce $3 \mathrm{D}$ reconstructions related to the history of a $\mathrm{CH}$ entity serving various purposes (for example, virtual reconstructions [3], various alternative scenarios possible in future, 3D animated applications for educational purposes, descriptive 3D models [4], or virtual anastylosis of fragments [5]) Some researchers have already focused on the importance of deciphering the buildings' life-time evolution [6], and the role of 3D modeling for the building's chronology. For instance, Reference [7] represents the dynamic evolution of $\mathrm{CH}$ assets in an effort to better understand the present status of $\mathrm{CH}$ according to its history.

Any 3D reconstruction, de facto, compromises a Level of Detail (LoD) according to the expressiveness grade of the geometrical models, systems, assemblies, or individual components. Nevertheless, a LoD does not extend to the amount of associated information, but only to the appearance of the geometry [8]. There are different classifications of LoDs in the literature [9], thus, we will adopt the scheme by Reference [10] in four grades, as follows.

- Level 1: basic outline as a solid object,

- Level 2: outline of the building as a solid object, with the principal and generic architectural features,

- Level 3: outline of the building represented as a solid object with all features, and

- Level 4: the detailed survey of the building represented as a solid object, covering all architectural custom components. Nevertheless, one should select the optimum level of graphical detail sufficient for the purpose considering time, cost, and effectiveness.

A comprehensive understanding of a historic building's chronology as such requires a representation in level 4 . However, we have set a modeling tolerance concerning the unavailability of information from the far-past, for not providing an incomplete model.

3D reconstruction acts as a data "container" for information regarding a historic building's chronology. This requires integration of geometric, non-geometric (for example, material usage, condition assessment), and external documents (for example, sources as historical texts or imagery). In this context, the concept extends to the Building Information Modelling (BIM) paradigm, which is based on the parametric modeling. This can be very relevant in providing some specific geometric information of architectural styles [11]. For the last decade, researchers have been targeting various steps, different shades of names (for example, heritage BIM, Historic Building Information Modelling, HBIM [12], BIM for heritage, and BIM for historic building [13]) and application fields from geometric data acquisition, along with the creation of unstructured point clouds to assist in the segmentation of the model into regions. This uses several segmentation algorithms or enrichment, based on different recognition techniques [14]. Nevertheless, we should highlight the fact that we will not focus on the ones which deal with automatic data capturing and point cloud integration phases. Rather, we will explore the capabilities in 3D model expressiveness, segmentation capabilities, hierarchical and relational model flexibility, and enrichment options for enabling time-dependent (that is, historical) queries. For instance, the work of Reference [15] focuses on mapping the decay in a BIM environment. However, it is limited to a surficial condition assessment rather than an object-based evaluation of the historic building. Besides, they report the complexity to graphically represent degradation pathologies within the BIM environment. The work by Reference [16] creates historic-building-specific libraries and assigns color codes to architectural elements differentiated by particular constructive phases. This study is thus, quite promising, but small in scale. This brings forth the question on the managing capability for capturing complex architectures. Similarly, the contribution by Reference [17] shows the possibility of BIM in $4 \mathrm{D}$ (that is, 3D plus time information) modeling the reconstruction of a defunct factory. However, the study is limited to the outer facade of the building. Both aforementioned studies do not extend to include the changes of architectural spaces in time; rather, they are focused on the building elements. Indeed, there are a few more promising approaches [18] offering time-based 
visualization and query possibilities for building elements. Nonetheless, the 3D elements in the model do not have a flexible hierarchical model which allows for spatial analyses (for example, exploring which space is transformed and/or altered rather than material properties).

A flexible hierarchy is important and based on which segmentation requirements and strategies are defined. Some studies offer automated segmentation possibilities, based on heuristics [19-21], context [22], ontologies [23], and prior knowledge [24]. Nevertheless, the identification of the different architectural components (for example, walls, windows, doors, and so forth) in 3D meshes is not enough. For example, if a wall is identified, it should be specified where this wall was connected, to which walls it was adjacent to, and which architectural space(s) it was hierarchically a part of [25]. Only in this way is it possible to define the mutual relationships of the components with each other in a hierarchical order. In addition, the building elements of historic buildings often lose their shape similarities over time due to deteriorations and alterations [15]. Thus, for the production of semantically enriched 3D models of any $\mathrm{CH}$ building, the use of automated segmentation algorithms or shape recognition is, in fact, not sufficient. Besides, the mutual and hierarchical relationships of building elements are either not considered or could not be applied, and the studies ignore the integration of non-geometric and external data.

Lastly, as far as the Geographical Information Systems (GIS) are concerned, they represent the most common approach for implementing the geometry/object and relation structure. There are still (some) prejudices regarding the GIS application to historic buildings, as the term "geographical" is understood as if it would only be used when referred to X, Y, Z coordinates. However, GIS is not bound to the creation of local or global geo-localization of elements. Rather, it refers to both the geometric and spatial relationships. GIS allows for geometric and non-geometric information modeling, where multiple relations between the data and (building) elements can be defined. The approach by References [26,27] thus focuses on discovering its potentials. In particular, Reference [26] integrates external information (raster or textual) to geometric information, whereas Reference [27] only focuses on the material deteriorations on the building's façade. It is, thus, possible to visualize and retrieve information through the 3D model, based on the building elements. Regardless, the geometric and spatial integration (that is, the relationships/hierarchies) between the building elements and spaces are not covered by both of them. More importantly, none of them cover the visualization for the building chronology or the related non-geometric information. Lastly, they only give access to the information through a single workstation by the use of specific software.

Quite recently, the reliability of these hypothetical reconstructions, their uncertainty grades, and the accessibility and transparency of the referred sources started to garner attention. We should note that these reliability measures do not have anything to do with the accuracy of the surveying [4], and are instead related to their correctness in relation to their corresponding sources. This is a critical issue, which is emphasized by the International Council on Monuments and Sites (ICOMOS) [28]. The full transparency of methods and techniques supporting a project of virtual reconstruction and the critical necessity to explicitly indicate information sources are highlighted, along with the need for alternative sources for comparison. It is underlined that complementary information gathered through prior research and investigation should be represented through the visual reconstructions, for the systematic analysis of both the architectural and historical data. This is a crucial problem, as many $3 \mathrm{D}$ reconstruction of historic buildings may not be fully documented despite containing a large, varied number of hypothetical, uncertain elements [29]. In uncertain visualizations, there is yet to be a validated and approved language, and researchers mainly use blurring or false coloring to depict uncertainty in their datasets. The contribution by Reference [30] points out the importance of the role that metadata and paradata play in modeling itself, along with the diffusion and archiving purposes. He underlines the importance of being "honest" about the process of visualization modality, ensuring the transparency of the sources as mostly possible. The work of Reference [31] adopts a 3D reconstruction strategy based on the uncertainty display and uses a variation of pseudo-color rendering objects into color bands, which corresponds with the level of uncertainty. 


\subsection{Potentials and Current Limitations}

Today, there is a great attempt to increase the BIM capabilities for more comprehensive, sophisticated, and query-able characteristics [32]. However, there are no applications that cover the characteristics of historic states yet. Besides, post-data processing for attribute enhancement and the integration of different datasets is yet unresolved. The Industry Foundation Classes (IFC), a standard data format developed by international Alliance for Interoperability, aims to cover both geometries and semantics [33]. Recent attempts for post-data processing and data management have been presented, both as open and commercial software solutions. Examples include the Building Information Modelserver [34], based on the IFC format, the Open BIM movement, initiated by Graphisoft, Tekla, and buildingSMART [35] for the enrichment of attribute data related to the building elements, zones, or complete buildings [36]. Nevertheless, the enhanced attribute management for historic building chronology is not yet an integral part of them. As such, the integration of external attribute related data is currently not fully covered. In addition, historic buildings necessitate an integrated representation of the different physical states. This requires a multi-layered modeling approach in which we would be able select a temporal state and represent it in a 3D environment.

Moreover, it is possible to create and access semantically-rich objects in a GIS environment, as proposed by Reference [33], particularly in the case of the semantic 3D city modeling process and urban conservation project assessment [37]. In this context, attributes can be easily joined to geometries and a relational database can be built in GIS. Indeed, the "standard" GIS products are still limited regarding its 3D editing functionalities as they generally only allow construction of simple $3 \mathrm{D}$ elements as geometric primitives. Thus, creating and visualizing complex 3D models of the chronology of historic buildings using a GIS software can still be problematic. In order to solve this, any compatible 3D modeling software can be used for modeling and coupled with certain plug-ins for importing/exporting. However, this will inevitably make the process longer. As an exemplification, we can create detailed 3D models in Autodesk 3DS Max and import them into a GIS environment with the use of plug-ins. However, in the conversion process, as the geometrical schemes and related descriptions are different and (sometimes) not compatible at all, some information would be lost during the translation, which means that particular attention has to be paid during this phase. While GIS products are still limited with regards to 3D editing functionalities, they show that there is a possibility to identify the key aspects for the representation of different information [38], enhancements with any type of attributes, and the querying or visualizing of any thematic concepts.

Consequently, many authors have started dealing with this topic, attempting a solution. However, an omni-comprehensive solution has not been proposed as yet. Among the existing approaches, there are only a number of projects that concern non-geometric and external data-sets. Nevertheless, heritage specialists need enhanced 3D models offered in a user-friendly environment, to allow for the fast and efficient information retrieval needed in the understanding of a building's history. This requires the storage of a huge-volume of geometric, non-geometric, and external data, and the capability to manipulate and manage them. Thus, in conclusion, information management applications in the field of $\mathrm{CH}$ for representing a historic building's chronological information are still in the early stages of development, due to multiple challenges. Today, it is possible to manage geometric data extensively in a 3D environment, but the enhanced attribute enhancement and integration of non-geometric and external data is not feasible. Correspondingly, there is no comprehensive research that directly supports the management of historic building's chronological information in 3D. In particular, a simple 3D model in a virtual environment for building chronology is not sufficient as it lacks any information about the historic building's internal structure, the hierarchical relations between the component building elements and spaces, and external sources.

\subsection{Research Aim and Content}

This research starts from a basic, yet important consideration: in each and every conservation-related action, a comprehensive understanding of a historic building's chronology as 
a whole is indispensable to be used as a basis for explicit reasoning and decision-making. Special attention is to be paid to the truthfulness of the information sources and the reliability of the model itself carries crucial importance, as it shapes one's understanding of a heritage building in both historical and cultural contexts. Correspondingly, the sources in diverse forms, (for example, drawings, photographs, written accounts) should be compared and adopted without leading to any speculative or unreliable information. Reaching definitions of the former time intervals while collecting information between the past and present, and attempting to understand the heritage building in a retrospective method of thinking, (that is, a scientific journey) has a comprehensive and overlaying form for both the mental and practical process for heritage scientists. An effective solution should cover critical evaluation and the truthfulness of records, and the synthesis of historic states showing (possible) changes of the building in time. In this context, this research aims at firstly, integrating time (that is, chronology/history) information into the 3D model, and by doing so, use a 3D model as an information container enriched with external (for example, textual and raster) data, in order to create an information management platform for efficient archiving, easy access, and long-term preservation.

Thus, in line with this vein of thought, this paper proposes an approach for the management of historical information of heritage buildings by creating a holistic 3D archive covering geometric and spatial aspects, along with historical information and sources (for example, annotating archival texts, old drawings, and the narration of travelers). Furthermore, this research has the motivation of easing access to the sharing of information and decreasing the future investigation efforts required in order to reach historic information of different periods.

The method of systematic conceptualization, the structuring and representation of information on 3D reconstruction, the production of complete geometric information, and its integration with non-geometric and external information into a complete and holistic platform is not a straightforward process (Figure 2). Rather, it carries systematic structuring rules which take the following concepts into consideration (a) segmentation: partitioning the buildings 3D geometry into meaningful sequences in terms of building elements; (b) structuring the hierarchies: setting the relationships between building elements and spaces; and (c) semantic enrichment: integration of historical information.

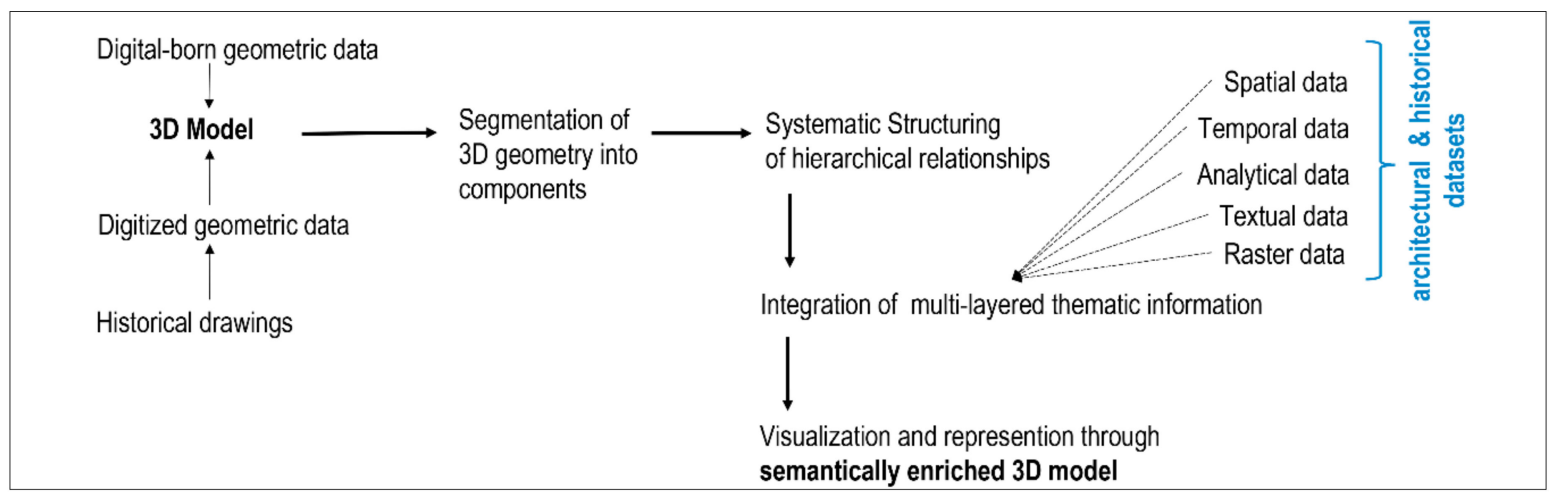

Figure 2. The process of creating semantically enriched 3D models.

\section{Materials and Methods}

\subsection{The Case Study}

The proposed methodology is implemented for the case of Kurşunlu Khan, in Manisa, Turkey. This is a typical building type of Ottoman architecture (şehiriçi hanı), dating back to the 15th century. In the medieval age, variations of the building with similar functions took different names in different geographies: caravanserai in the Middle East, gasthof in Germanic, the inn in Anglo-Saxon, hospitium or auberge in Latin, and fondaco in Geneose and Venetian [39]. The geometric order of Kurşunlu Khan 
is derived from the Ottoman classical style and the volumetric order consists of two floors that are almost square schematically, with a central courtyard surrounded by a colonnaded portico.

The khans were among the irrevocable components of Ottoman town centers, which were not only a place for exchanging goods, but also a place for craftsmanship activities with many workshops. Alongside that, the buildings during the Ottoman Empire were crucial in representing the image of the town. Thus, Ottomans reshaped towns and built several institutions and monuments as markers of their civilization. The town centers were important nodes that narrated urban culture and an evaluation of the political and socio-economic structure of the Ottoman Empire showed that the construction process of building complexes and its profit-earning components, such as khans, were part of the government strategy. Accordingly, the building itself is a representative of the Ottoman urban life in Manisa, that is, it implicates many values representing the architecture and technology of that period, along with its culture and economy. The building is an essential component that contributed to the image of the townscape while the life of the historic setting of Manisa is considered. As a consequence, Kurşunlu Khan also has documentary value, as a type of construction of traditional culture, architecture, and land-use. As a matter fact, the building is a form of the Ottoman culture and lifestyle, which represents a genuine dialogue [40] of the Ottoman era. It is a physical reality, as a result of a creative process of mankind [41].

In the architectural scale, Kurşunlu Khan (Figure 3) kept its original function, which was built before the industrial revolution and its inadvertent impacts: the changes in the urban pattern and commercial center, and the confrontation of the Ottoman craftspeople and merchants with it. It was found out that the Jewish community was residing there in the 16th and 17th centuries, and it housed a cereal market in the 16th century. While having minor and major repairs in 1611,1643, 1677, and 1848, and some interventions due to both man-made and natural causes (covering the 1st, 2nd, and 3rd states), it continued accommodating the original material culture. The re-functioning of the khan as a prison in 1908 (4th state) created a dramatic change both in its function and in its spatial composition, including the conversions of semi-open spaces to closed ones, and the removal of various openings. Following that, in 1954 (5th state) the widening of the Borsa Street caused a slight change in the mass volume, which was caused by the demolishment of 11 shops in the Northern direction. In 1966 (6th state), the restoration campaign was intended as a positive contribution for covering the building's structural deficiencies and static weaknesses. However, the choice of concrete as a restoration material was inappropriate. This was followed by an unintentional intervention while the building was converted into a student dormitory (7th state). Yet, the current restoration campaign aims to fulfill the requirements of an adequate conservation [42]. Consequently, the physical structure of the Kurşunlu Khan has undergone a series of alterations, transformations, and conversions, but major parts of the building's structure could be preserved due to its durable construction materials.
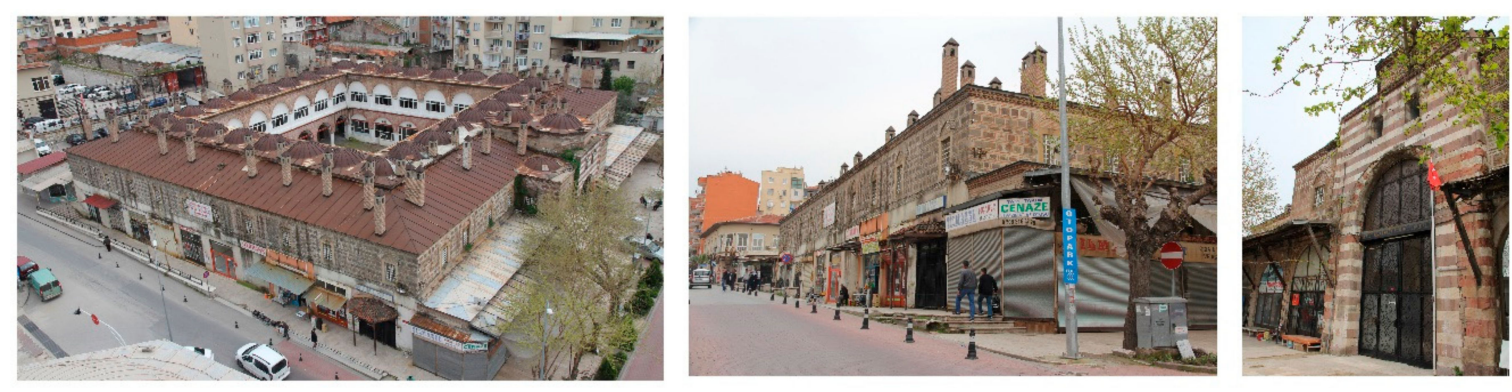

Figure 3. The Kurşunlu Khan as seen today.

\subsection{Conceptual Modeling and Design}

Considering the peculiarities of historic buildings, the information management systems helps to separate segments of information and relink them together in a structured way which allows new 
information to be derived, or to aid its link to 3D data [43,44]. This aid would come in the form of the semantic enrichment of 3D models, which is also a potential solution for archiving historic building's chronological information. The criteria for its production process needs to include: (i) the definition of mutual and hierarchical relationships, (ii) attribute management, (iii) 3D editing functionalities, (iv) spatial and multi-criteria query-able characteristics, (v) representation of datasets in 3D, and (vi) the representation of different restitution states. Regarding the defined criteria above, it is necessary to go beyond the geometric data which is composed of either 3D or 2D geometries.

The conceptual modeling starts with the abstraction process. The identification of the "seen" and the classification of the objects is closely related to the "reading" metaphor for the architecture of any building. Any description of an architectural property has double-sided aspects: solid and hollow components [45], which come together to produce the "wholeness" of human perception. Therefore, the components that exist are as follows: firstly, the building element as solid features and secondly, the architectural spaces as hollow ones. The former is a mathematical construction of physical components which have roles (functional, constructive, and so forth) and morphologic types. The latter is born from the specific relationships among the former and has explicit limits created by them. Thus, the spaces are identifiable as different hollow volumes that one can interpret by following a cognitive process, as they are not physically solid geometries (Figure 4).

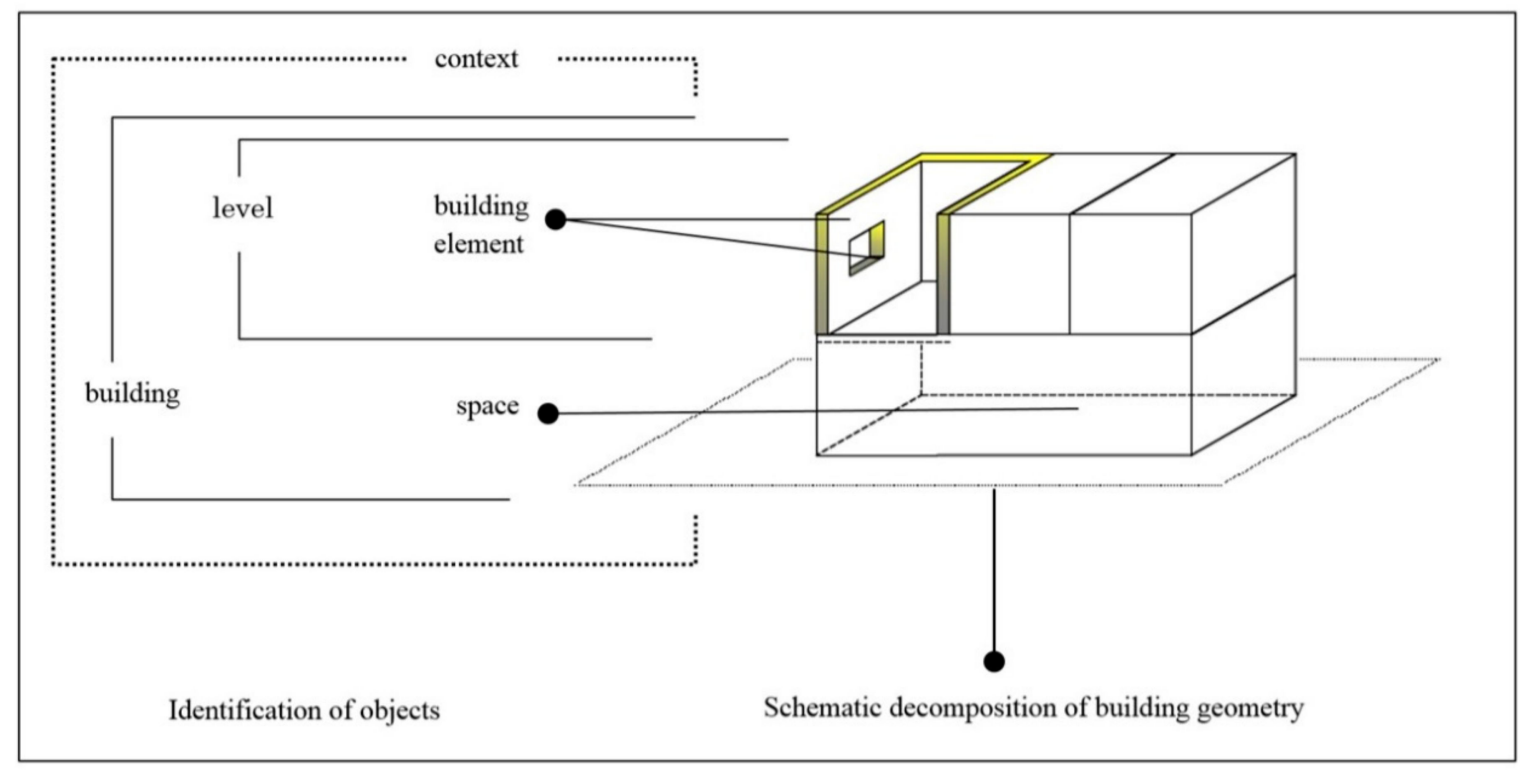

Figure 4. The reading of a building and the identification of features.

Within the lighted area of the above-mentioned identification process, the conceptual modeling is carried out for the case study, in order to perform its systematic decomposition. In this process, the relationships among the different building parts, along with its hierarchies, are defined. Figure 5 represents the conceptual model prepared for the case study. A standard graphical notation and vocabulary are used, thanks to the Unified Modeling Language (UML) [46]. UML is a language which helps with the representation of conceptual schemas graphically. The conceptual schema is independent of any specific architecture (for example, software, database system, and so forth). The diagrammatic representation of a class, for example, is displayed in a rectangular form, and it includes three sections: the top section, which gives the class name; the middle section, which includes its properties (for example, attributes); and the last section, which includes (optionally) operations associated to this class.

The Object-Oriented $(\mathrm{OO})$ modeling approach is adopted to define classes, their properties, and the relationships (associations) among them. The building elements that have identical characteristics are grouped together to form classes (that is, abstract objects) which share common 
properties. Another step is to identify the way classes are connected to one another through links, thus, setting particular hierarchies and assigning different associations. For example, the class of "space" is an aggregation of the classes of "wall", "window", "door", "slab", "dome", and so on.

After completing the conceptual schema, an entity-relationship (ER) model was manually derived for the successive database implementation (in this case, for PostgreSQL/PostGIS). For the creation of the PostgreSQL database schema (Figure 6), all the required database objects (for example, schemas, tables, constraints, data types, and so forth) were created. The reason for adopting the PostgreSQL relies on an advanced open-source database that works with the relational database management concept [47] and also on the availability of its spatial extension of PostGIS. This allows the use of special data types for geometric objects and enables their storage, along with their manipulation and querying. The capability is not limited to only $2 \mathrm{D}$ primitives, but also allows for $3 \mathrm{D}$ geometries in the form, for example, of polyhedral surfaces and triangulated irregular networks (TIN). Besides, PostGIS supports both raster and vector data types and it conforms to geographical and spatial data standards of the Open Geospatial Consortium (OCG) [48].

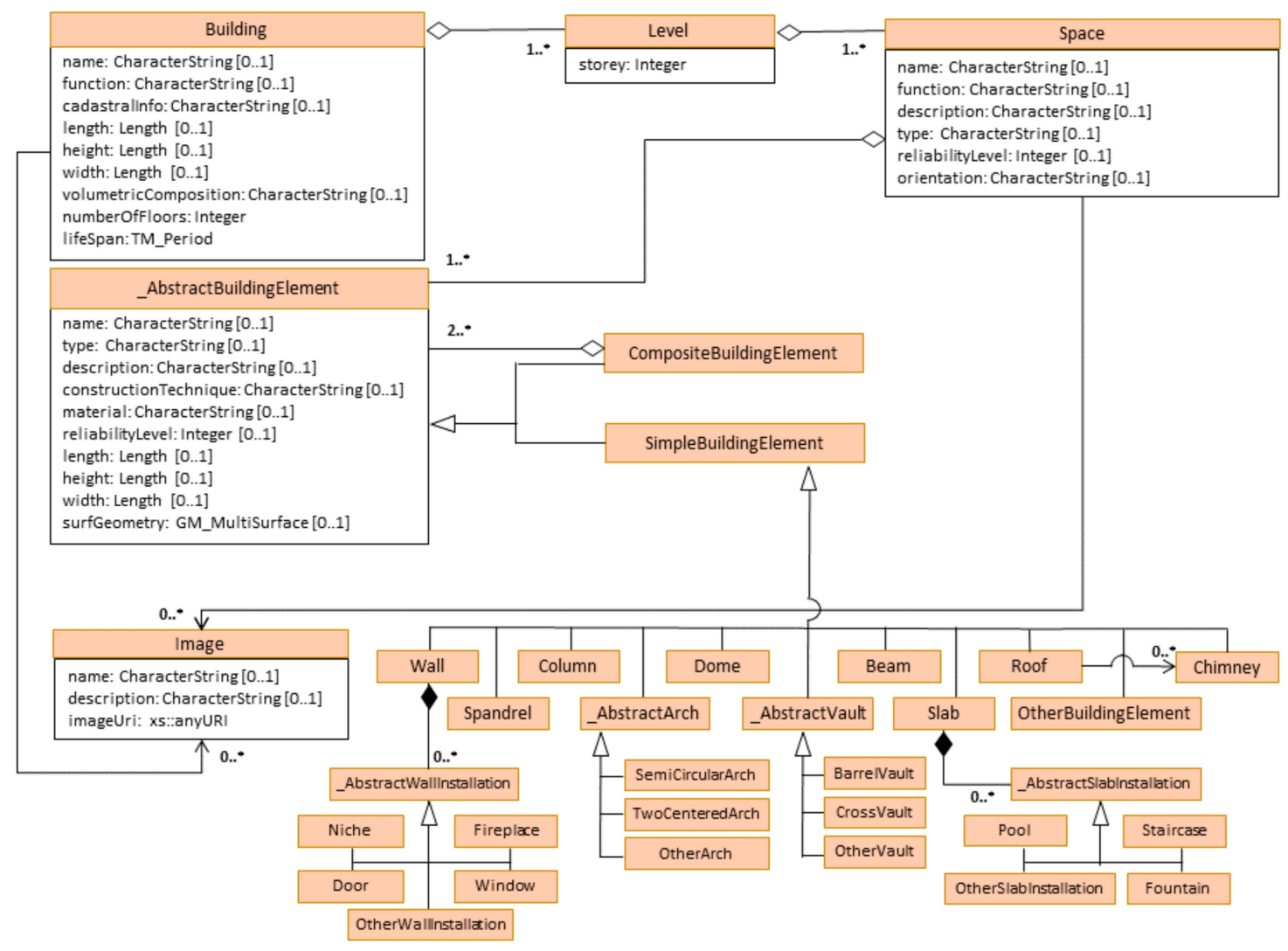

Figure 5. The Unified Modeling Language diagram for the case study.

\subsection{Workflow of the Process}

The proposed information management system should allow for the archiving of a semantically enhanced 3D model for each historic state within the building's chronology in a flexible way, and should also be capable with the assigning of different data for its diverse parts and components. The process, the related tools, and software are illustrated in Figure 7.

It should be noted that, in the case of Kurşunlu Khan, a static system of representation is applied in the description of the temporal phases presented here. This is the result of determinate changes of the building elements or spaces in particular historical moments. Uncertain or poorly documented changes are, however, not covered as restitution states in this work. For instance, in the case of a repair 
campaign or minor interventions with unspecified building elements or spaces, cases where they could not clearly determine both the physical aspects (space) and specific moment (time) were neglected.

\subsubsection{Preparation of the 3D Model}

Currently, architects use 3D modeling as a standard practice for an efficient visual representation of the buildings. Unlike approaches based on on-site data collections, via either photogrammetry or laser scanning with the results on the digitally generated 3D material, this study makes use of readily available 2D information. The available graphical documentation is in the form of the architectural plans, sections, and elevations, which is a standard way for representing architectural heritage information. The representations cover two major data-sets, the measured drawings, and the restitution drawings. All these are available in the form of 2D CAD drawings [42], which are composed of geometric primitives and textual annotation. Nevertheless, moving towards 3D models from 2D drawings does not only involve the creation of volumetric objects, rather, it is a more complex process. In the present day, the generation of 3D models from 2D drawings can be automated [49,50], semi-automated [51], or manual [2]. Whatever the method applied, they all require a significant manual pre- and post-processing effort [52]. In particular, for historic buildings which are composed of complex geometries and lose the similarities of their shapes on an object level, the manual method is mostly required.

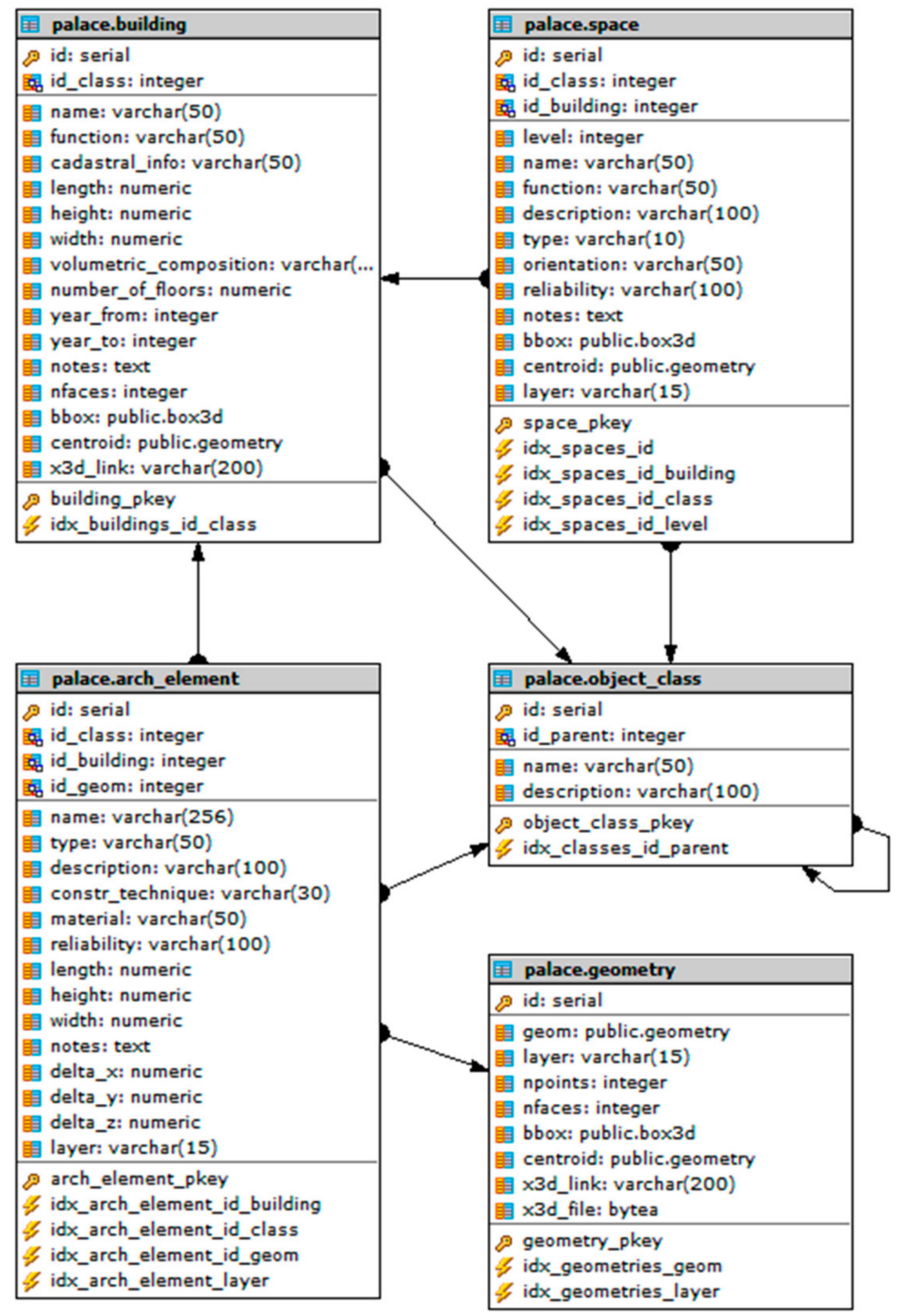

\begin{tabular}{|c|}
\hline 围 palace.relations \\
\hline id: serial \\
\hline $\begin{array}{l}\text { 国 child_table: varchar(30) } \\
\text { 用 id_child: integer } \\
\text { 国 relation: varchar(30) } \\
\text { 用 parent_table: varchar(30) } \\
\text { 国 id_parent: integer } \\
\text { 闻 notes: text }\end{array}$ \\
\hline $\begin{array}{l}\text { Drelations_pkey } \\
\text { idx_relations_child_table } \\
\text { idx_relations_id_child } \\
\text { idx_relations_id_parent } \\
\text { \& idx_relations_parent_table }\end{array}$ \\
\hline
\end{tabular}

\begin{tabular}{|c|}
\hline 国 palace.image \\
\hline id: serial \\
\hline 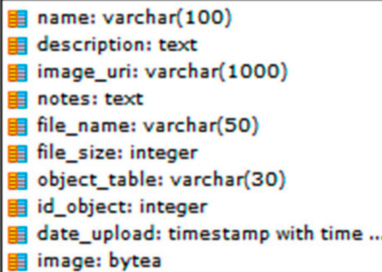 \\
\hline $\begin{array}{l}\text { Dimage_pkey } \\
\text { images_id_object_idx } \\
\& \text { images_object_table_idx }\end{array}$ \\
\hline
\end{tabular}

\begin{tabular}{|l|}
\hline palace.external_data \\
\hline id: serial \\
\hline 国 file_name: varchar(256) \\
闻 description: text \\
国 notes: text \\
国 file_extension: varchar \\
国 file_path: varchar \\
国 file_size: integer \\
国 file_link: varchar \\
国 file: bytea \\
国 upload_date: timestamp with time ... \\
国 object_table: varchar \\
国 object_id: integer \\
\hline external_data_pkey \\
\hline
\end{tabular}

Figure 6. The database schema implemented for the study. 
As the 2D CAD drawings cover only the building's layout, they lack details and 3D spatial descriptions needed for holistic representations. The process of 3D model generation from 2D drawings is thus, an effectively possible method to represent any spatiotemporal state. Nevertheless, this could prove to be challenging as there are only a limited number of section drawings, which, in turn, creates a lack of depth in the information for certain part of the spaces. When visualizing in 3D, one starts to find out where there is a pre-existing gap of knowledge. Eventually, the manual methodology for the geometric modeling is derived through the use of computer-aided architectural design (CAAD) methods, as is adopted for the Kurşunlu Khan. Through this, it thus aims to represent a LoD in which a vital understanding of the building will be offered without exhaustive details.

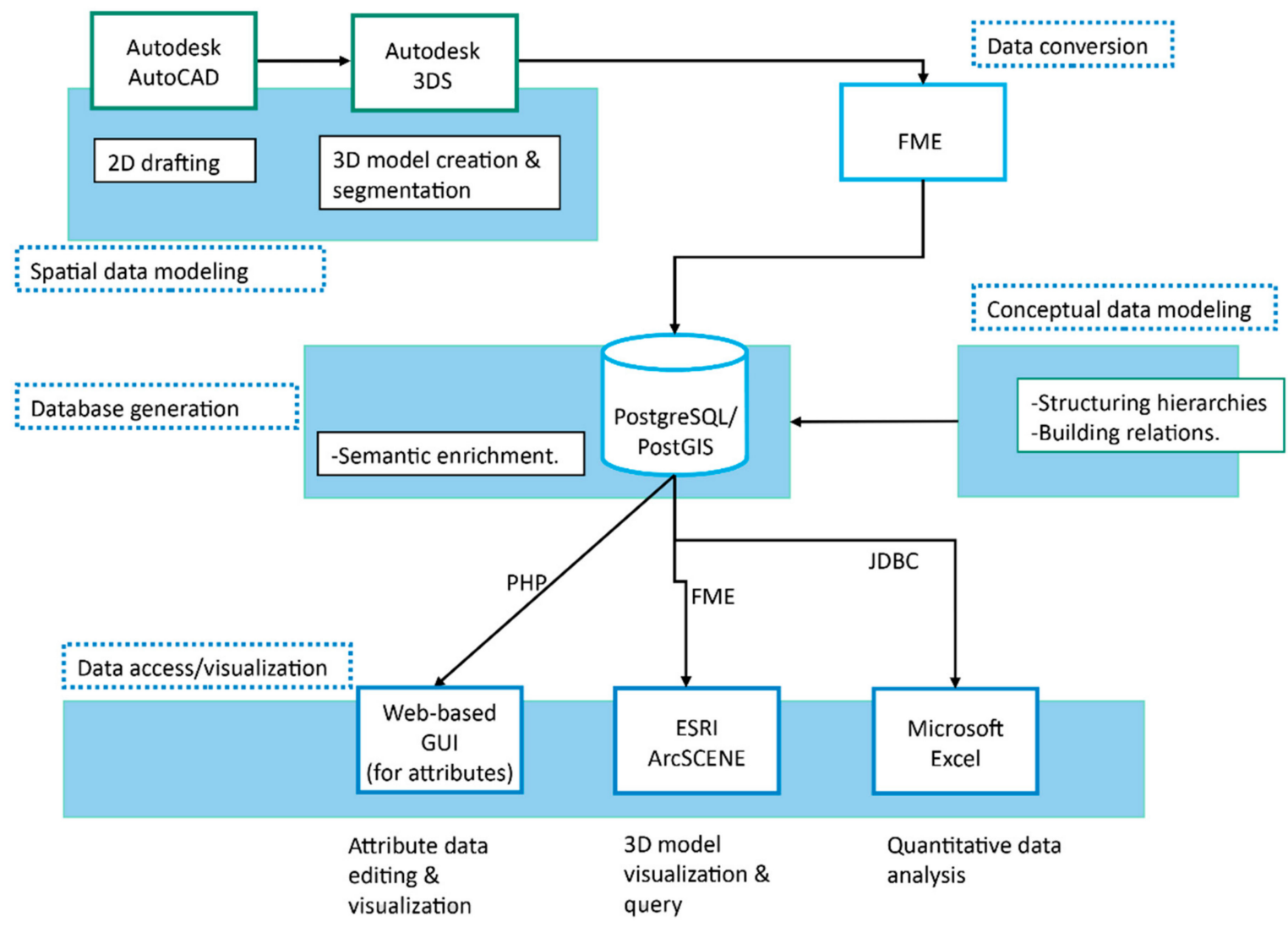

Figure 7. The overall workflow and architecture.

A deductive approach is considered for the logical segmentation of the Kurşunlu Khan, with correspondence to the classes and relations as defined in the conceptual data model. However, an inductive approach was applied to the building, as assigning different datasets included textual, numeric, vector, raster data types to objects and classes (Figure 8). Consequently, the creation of the segmented 3D model has been a process of reverse engineering. The model is drawn from the smallest segments upwards, in a bottom-up approach, thus, allowing for the assignment of thematic datasets of building chronology to the objects of the 3D model. 


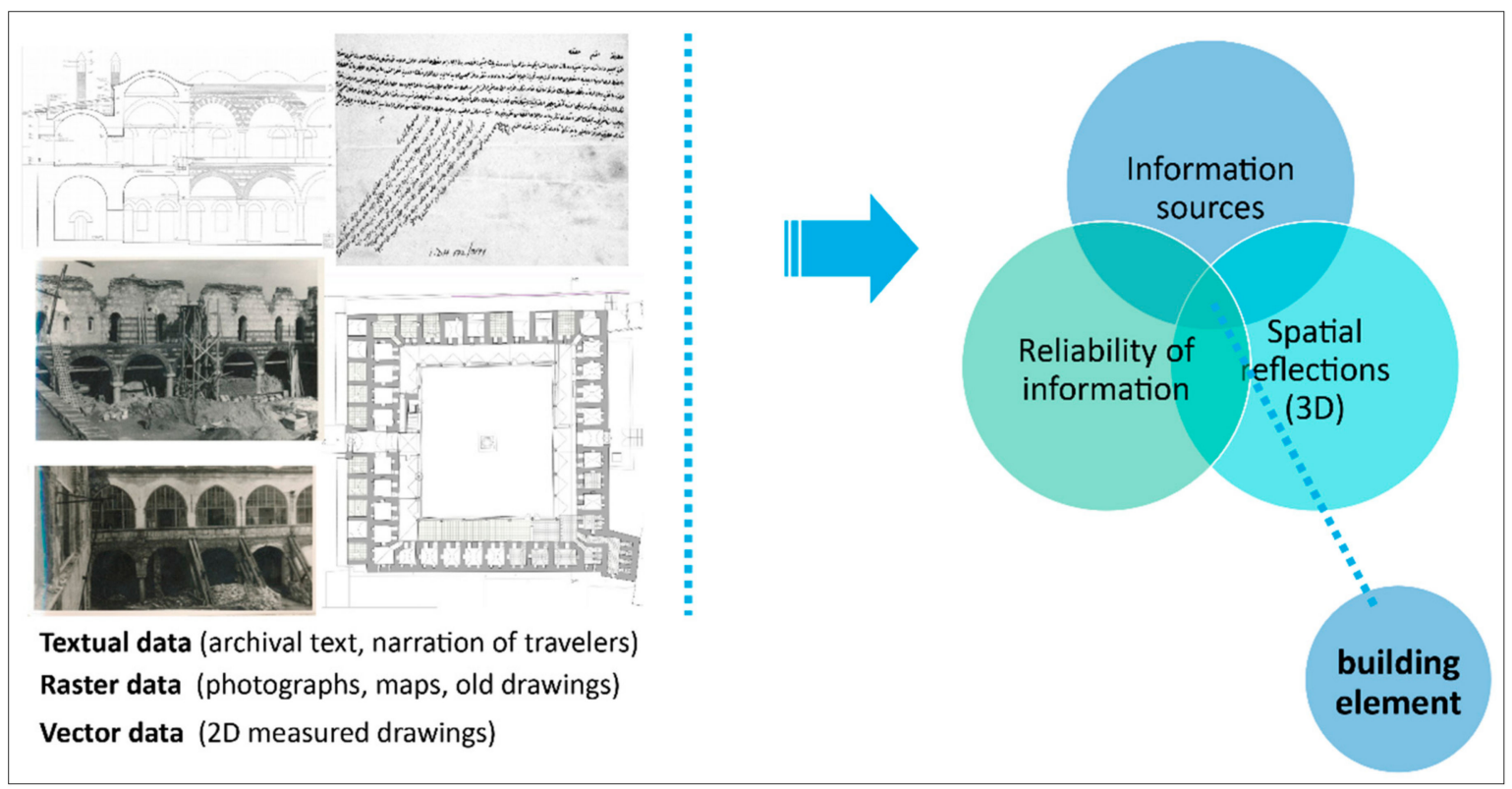

Figure 8. The abstraction of the inductive approach applied for assigning different datasets.

Autodesk 3DS Max is used as the primary 3D modeling tool (Figure 9). In the overall process, the aim was to produce 3D geometries that represented the different architectural elements. Accordingly, closed polygons and solid features are used. Essentially, 3D modeling was started with the extrusion process of vertical components (for example, walls) using a 2D floor plan created in AutoCAD. As such, it is not a straight-forward process and considerable effort must be paid to the following issues: (i) the segmentation decision for overlapping or joining edges, (ii) the differences in height information on a single object, (iii) openings that are in non-rectilinear forms need to be subtracted.

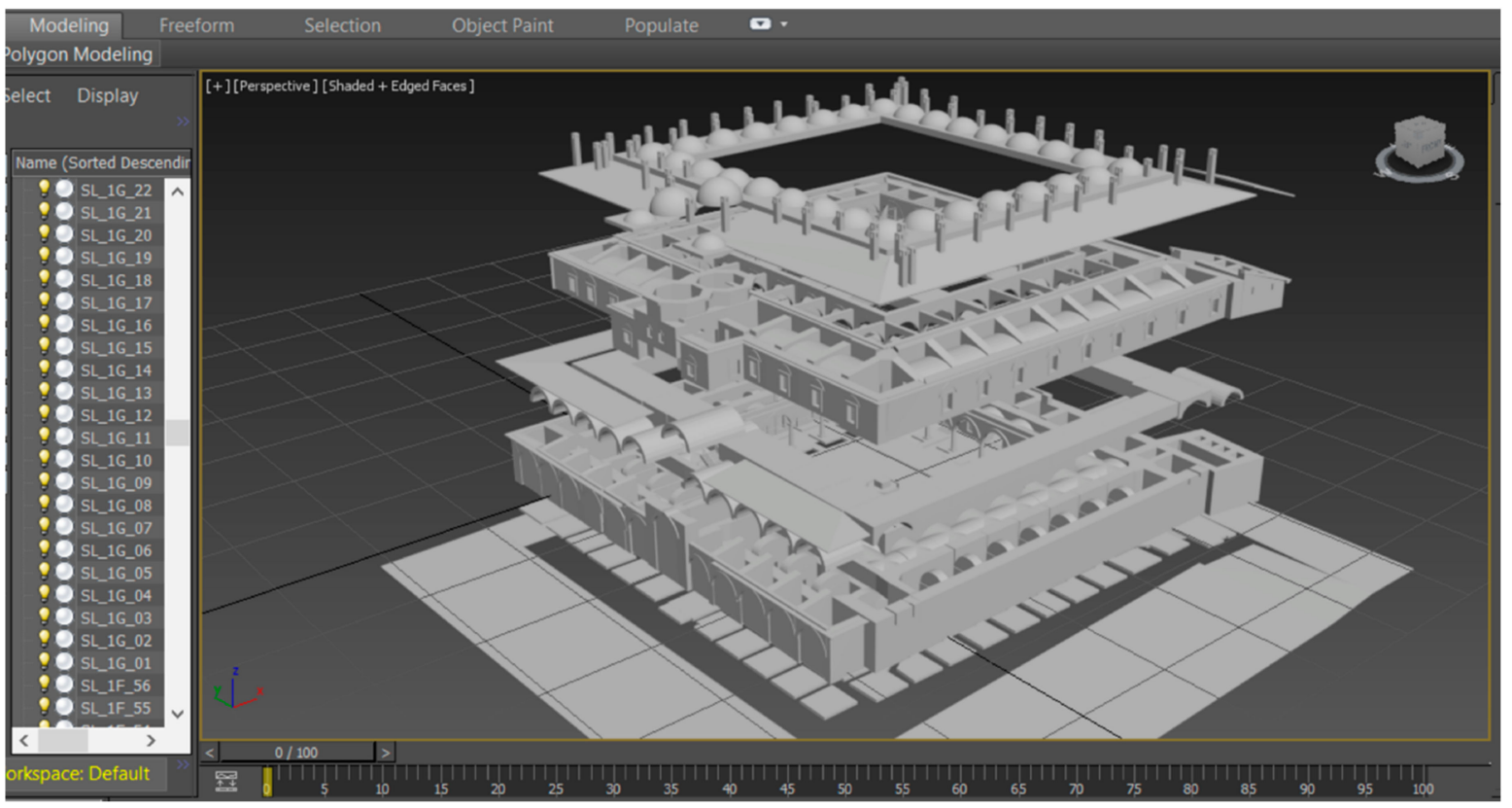

Figure 9. Assembling the segmented 3D model in Autodesk 3DS Max.

Another step involves the modeling of geometries, which cannot directly be created by the extrusion of planimetric information. Building elements, such as arches, vaults, domes, spandrels, 
columns have to be modeled individually in correspondence with their respective sub-classes. Nevertheless, modifications are done for each individual object in order to cover all the diversities, that is, changes in dimensions (height, width, diameter, and so forth), along with the inclusion of specific alterations.

Thirdly, horizontal components, in the forms of slabs, are considered. The decision of the detection of the boundaries of each one is done in correspondence to the building's element-space relations. Lastly, other building specific elements and installations, such as doors, windows, chimneys, moldings, and staircase are modeled.

In 3D modeling, the goal is to characterize each building element with a specific name and function. This is in order to place it conceptually into the hierarchical tree diagram. In this case study, a naming convention was applied as a viable solution for the assignment of each corresponding object. This was done by means of the standardized name coding, due to the scarce capabilities in Autodesk 3DS Max to assign attributes to the geometries. Hence, during the use of other software, one may have to adopt other strategies in order to have the geometry classified.

It is possible to regenerate a segmented 3D model for any restitution state. The building elements which remain original (unchanged), from one state to another, are preserved in the 3D model. Thus, the original building elements remain unchanged, while the modified ones are removed. Correspondingly, the original ones will continue to hold the same naming convention and newly added geometries will have new unique values based on their properties. It should be kept in mind that this rule can only be applied to the geometric modeling. When it comes to attributes, it is possible to assign different attributes to the same building element in different restitution states, despite the geometry not changing.

The geometric modeling process is followed by the translation process, as performed by the Feature Manipulation Engine (FME) by Safe Software Inc (Surrey, BC, Canada). [53]. The FME Workbench ETL (extract, transform and load) tool provides an efficient and semi-automatic feature manipulation, with a user-friendly graphical user interface, in order to visualize the data-flow. Consequently, geometric data in the .3ds format is read (extracted), transformed, and written (loaded) without any losses; for example, from 3DS Max to the PostgreSQL/PostGIS database, or from the database to a shapefile, or to any other common format.

\subsubsection{Semantic Enrichment}

Once the 3D objects representing the different architectural elements have been created, imported into the PostgreSQL database, and further grouped to generate the corresponding higher-hierarchy objects, (for example, spaces) they are enriched with attributes using the following criteria.

Firstly, in the "building elements" table, the element name (arch, vault, wall, and so forth) and the element type (structural, architectural or finishing) are included. Those are followed by the attributes belonging to the construction technique and material usage (cut-stone, brickwork, stonework, and double color broad-stone with brick courses, and so forth), along with information regarding the element size (width, length, height), which are also indicated. It should be noted that these are not the exact measurements of the elements, but of the bounding boxes defining each of them. Further attributes, such as the reliability levels, are included. The reliability level represents the different degrees of the hypothesis' truthfulness of the geometric feature it refers to and is expressed with values ranging from the 1st to 3rd level. The sources from the various mediums (for example, drawings, photographs, written accounts) are compared and only adopted in use if it corresponds to the criteria of being non-speculative and reliable information. To understand this using an example, old photographs are sources with 1st degree reliability, as they are non-speculative records. Historic dates of the administrative documents coming from Ottoman archives can also be viewed as primary sources for noting the changes. However, as the content of these changes are not specified in detail (location, material, size, form, and so forth), they have a 2nd degree reliability level. 
Populating the "spaces" table is a manual process. The attribute values of the level can be, for the Kurşunlu Khan, 0 or 1, representing the ground floor or the first floor consequently. The function attribute holds values, such as the room, portico, courtyard, lavatory, and so forth, and the spatial type is defined by three possible values: open, semi-open, or closed. In addition, the values of the geographic orientation can be North, South, East, or West.

For populating the "building element-space", the very first temporal state has to be manually inserted individually for each segment. Furthermore, special attention must be paid as some segments might be shared, for example, a wall facing towards two adjacent spaces belonging to both of them. As a consequence, two rows with the same building element name can appear. In such cases, that element is a shared one and used for shaping more than one space due to the hybrid approach used.

After populating the building element-space table for the original state, it is possible to continue with the other temporal states, in chronological order. We keep the idea of re-using the 3D geometry of a building element, as it might remain the same in different temporal states, thus, showing that it remained unchanged. In this case, it can be cloned and reused. Indeed, it is also possible that it could form different spaces in different temporal states due to any possible spatial changes. As an example, a column might be part of the colonnaded portico as a semi-open space in one state, whereas in another state it might make up a room due to some conversion. As a consequence, it is only possible to clone and reuse relations within the building element-space table of consecutive temporal states only for spaces that did not have building elements removed or include the addition of new elements. In cases where space has undergone any type of change (transformation, removal, conversion, and so forth), the new relations have to be set manually.

In the building's table, the different temporal states are inserted as different buildings. This allows a representation of the chronological changes in the function of the building, such as mercantile, accommodation, prison, religious education, and dormitory. Volumetric composition, cadastral info, and dating information are populated with the information coming from historical research.

\subsubsection{Data Access and Visualization Options}

Data visualization enables decision makers to see analytics presented visually so they can grasp difficult concepts or identify new patterns. With an interactive visualization, one can take the concept a step further, by using technology to drill down into charts and graphs for more detail, interactively changing what data you see and how it is processed.

In this work/approach/proposed solution, it is possible to export data to ArcGIS and visualize them in 3D, using certain software such as ArcScene. Through this method, it is possible to do a comparison of different restitution states and their reliability levels and gather a critical interpretation. In Figure 10, the consecutive restitution states and their reliability levels are visualized in ArcGIS. Colors and transparency are used for easy detection. In particular, image in Figure 10c shows the 3D visualization of the restitution of 2nd State (1643), while the red in Figure 10d represents the 2nd degree reliability covering the repaired building elements following the earthquake; in particular, this relates to the 12 arches on the ground and the first floors in the Southern direction, along with the windows and doors of the rooms. Additionally, the roof cover of the stable is shown in purple, thus representing a 3rd degree reliability. In Figure 10f, the red again represents the 2nd degree reliability, covering the changes due to a repair campaign following a previous fire; in particular, this includes the replacement of the roof covering, the addition of the drums below the domes, the reconstruction of the shops on the West with a longer depth, and the modification of the Western portal. Figure $10 \mathrm{~g}$ is the visualization of the restitution of the 4th State (1908), and below, in Figure 10h, the red again represents the 2nd degree reliability covering the interventions due to the re-functioning of the khan as a prison; in particular, the transformation of the colonnaded portico into rooms by adding walls, doors, windows, and the addition of a wooden shelter in the courtyard. Figure 10i is the visualization of the restitution of the 5th State (1954), below in Figure 10j, with blue representing the 1st degree reliability, and covering the intervened parts; in particular, the demolishment of the shops, the removal of a room, and the 
addition of a new gate, changes in the openings and application of plaster, all at the Northern direction. Figure 10k is the visualization of the restitution of the 6th State (1966-1970), and the blue in Figure 101 represents the 1st degree reliability covering the interventions of the restoration works; in particular, the reconstruction of the colonnaded portico, including its components: columns, vaults, domes, and so forth; the reconstruction of the shops located on the West; the replacement of the casings of windows and doors, replacement of the roof covering; and reconstruction of chimneys.

In addition, it is possible to do queries of the semantically enriched 3D model. In this context, ArcGIS allows for two ways of query-based visualization: firstly, selection through attributes for visualizing within the complete model, and secondly, by using the query builder to solely visualize the corresponding building elements. In addition, it is possible to do queries according to the building element name and type, material usage, element size, restitution state, and its reliability. In order to exploit different query possibilities, some conservation-aimed examples are illustrated below. As an exemplification in Figure 11, only the hypothetical building elements, which are classed as the drum by element type and have the 1st degree of reliability, are queried and visualized at the reliability of restitution model in the 3 rd state.

Other than the above-listed possibilities, it is also possible to click on an element and gather specific information on that element, based on the associated attribute tables. Furthermore, it is possible to visualize images which are assigned to the nearest building element as a source of restitution for a better understanding. As an example, in Figure 12, the identify option is applied to a building element within the colonnaded portico in order to understand how that space was converted. This allows both the information on that building element and archival photograph belonging to the colonnaded portico to be discovered.

Another data access possibility is offered through a web-based platform, due to a PHP-based web GUI, which allows a common and user-friendly interaction with the PostgreSQL tables. Accordingly, it provides a web-based browser interface that gives the possibility to visualize, edit, and export tabular data from the database (in csv, pdf, and xls). The 3D model of each restitution state (Figure 13) can also be exported as an X3D format to be visualized in external viewers (for example, in Meshlab [54]). The X3D files, however, only contain geometries, that is, no semantics. Furthermore, a pull-down menu allows the user to reach tables of other significant data, (for example, images) which are linked to the corresponding state of the building chronology (Figure 14). In addition, on the main page, there is a tab titled "external data", in which sources of information, published and unpublished accounts, descriptions, and other archival documents are included. This also gives the possibility to individually view and edit each item as well. 


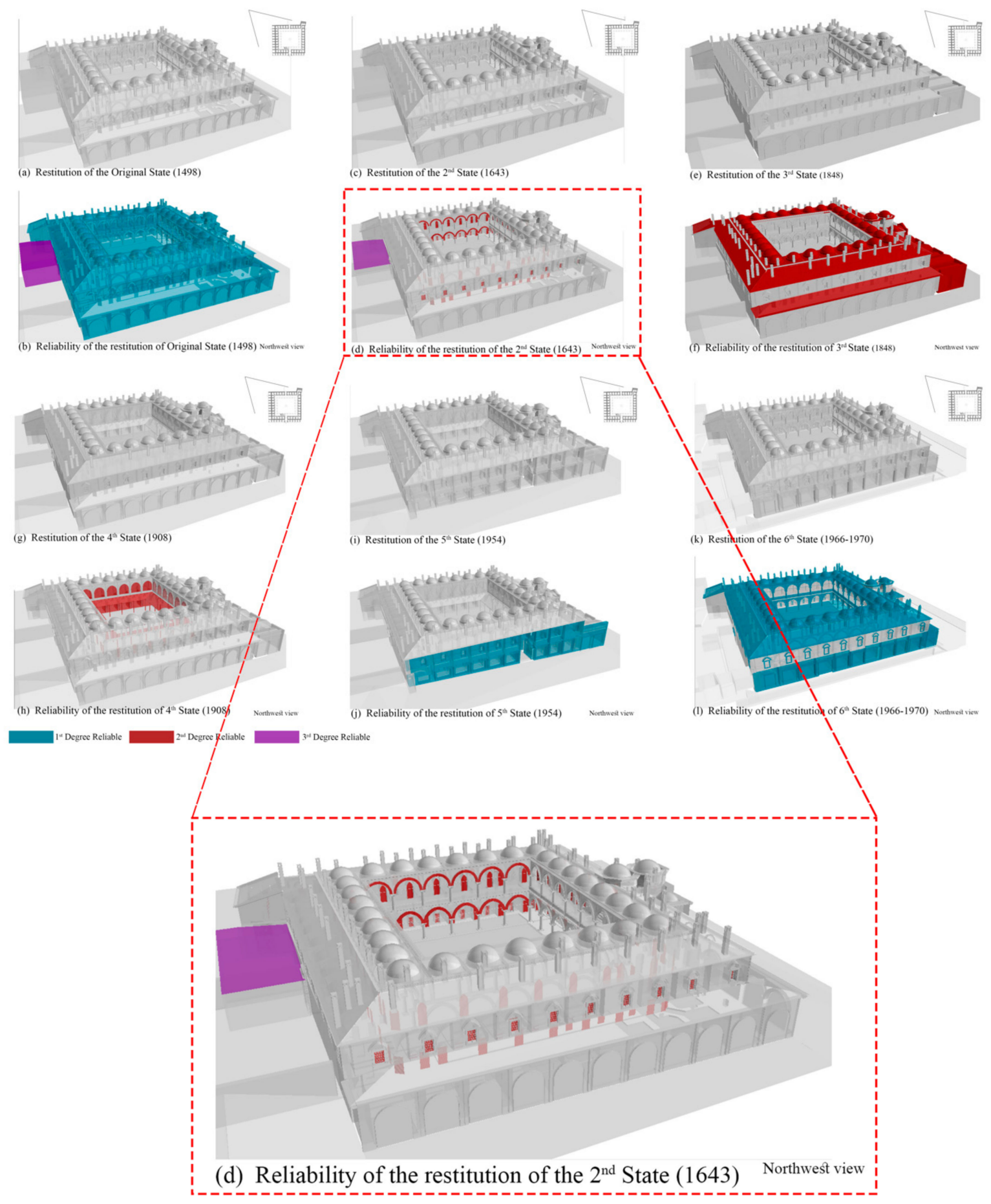

Figure 10. The visualization of consecutive restitution states (1st to 6th) and their reliability levels (1st, 2nd and 3rd degrees) with color-codes assigned for easy recognition.

Finally, it is possible to export data from PostgreSQL to Microsoft Excel by means of the pgJDBC (Java Database Connectivity) interface, in order to exploit its well-known data analysis capabilities and functionalities for creating reports and generating graphs or charts. However, it should be noted that this would just be a method of quantitative comparison of either building elements or spaces according to the different parameters. Nevertheless, a historic building requires complementary qualitative analysis for a full understanding and some data extraction possibilities are provided. The aforementioned are not aimed to cover every notable aspect (Figure 15). 


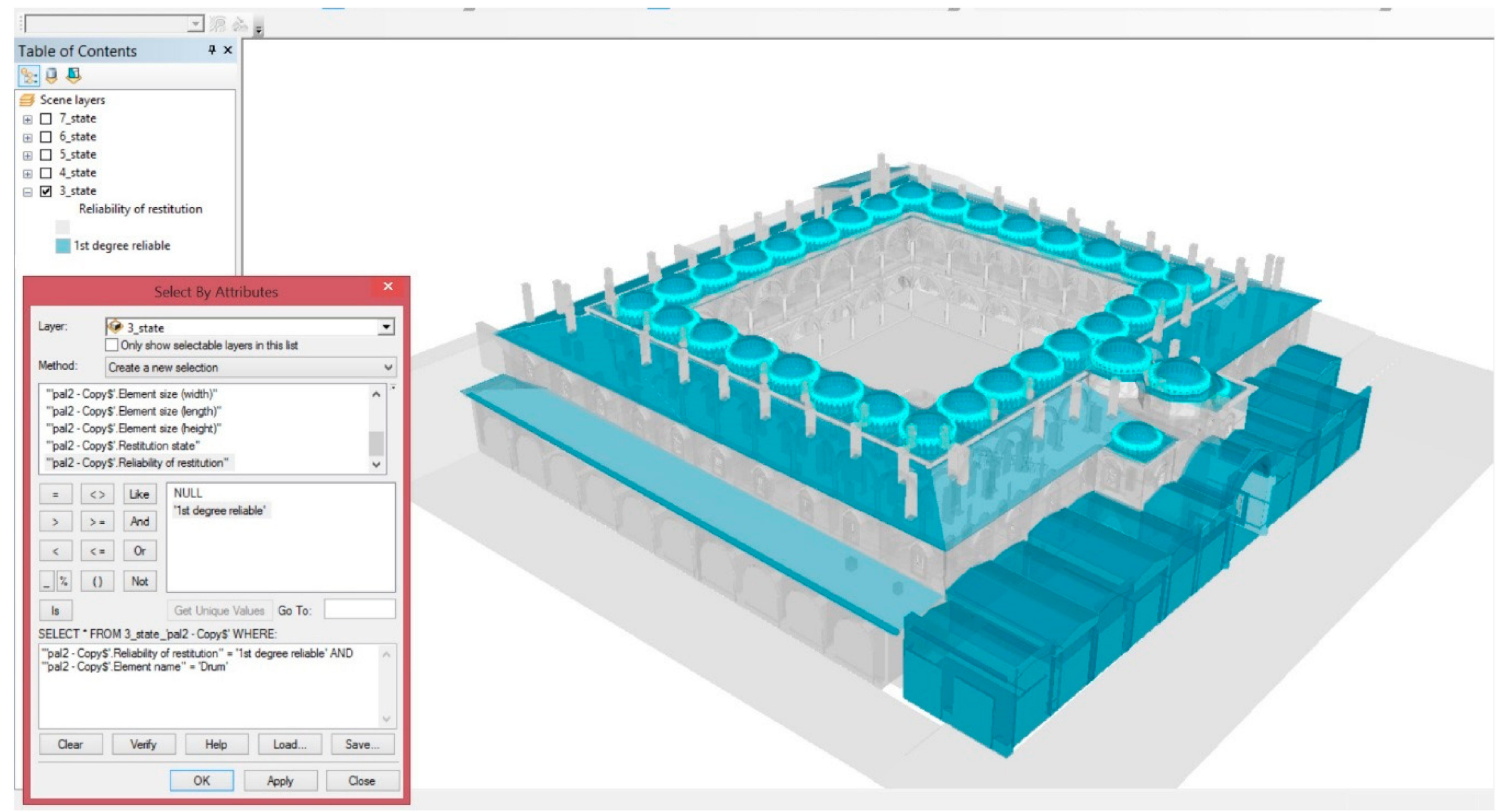

Figure 11. The query of the 3D model (3rd state) in ArcScene. 


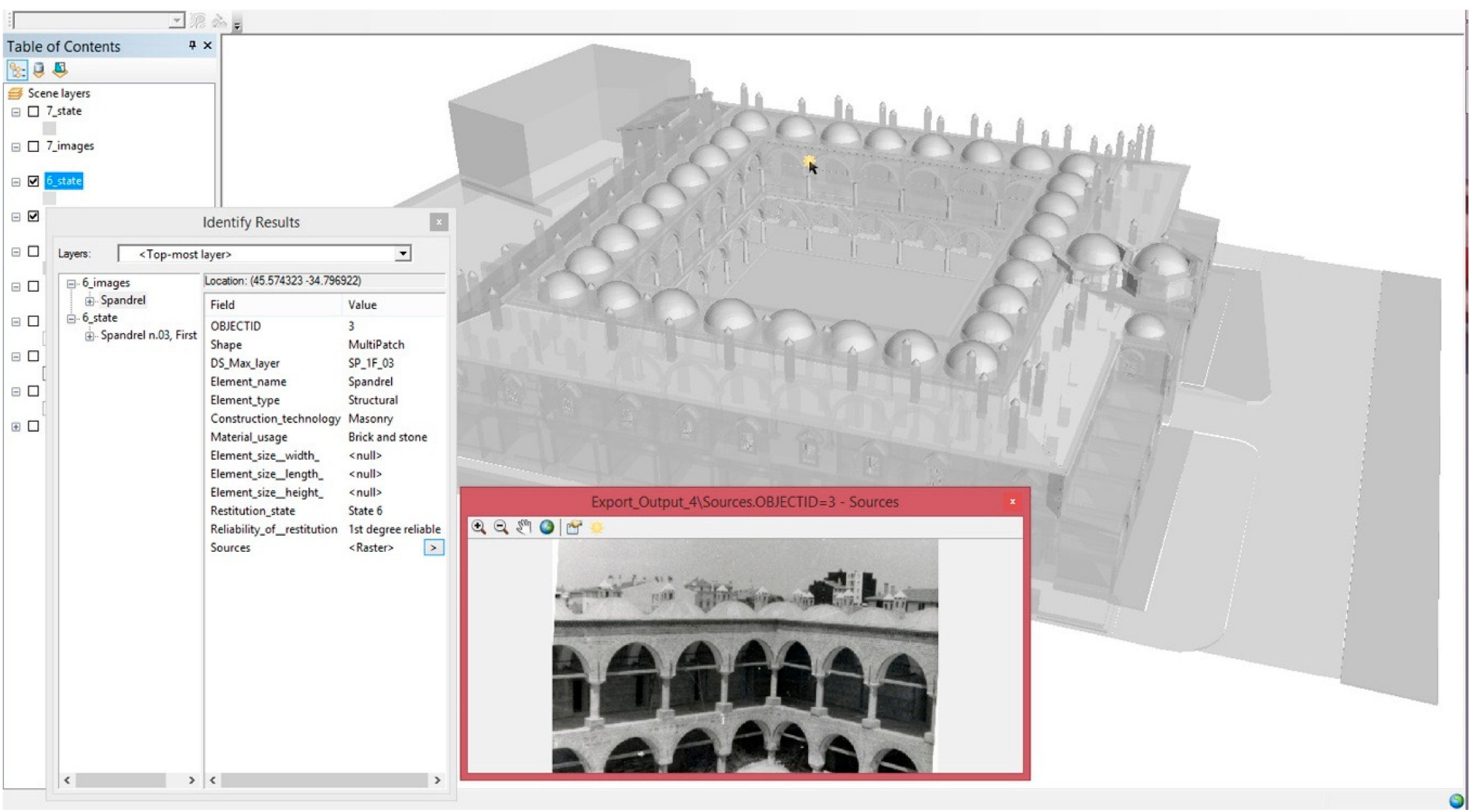

Figure 12. The application of identifying the options through the 3D model in ArcScene. 


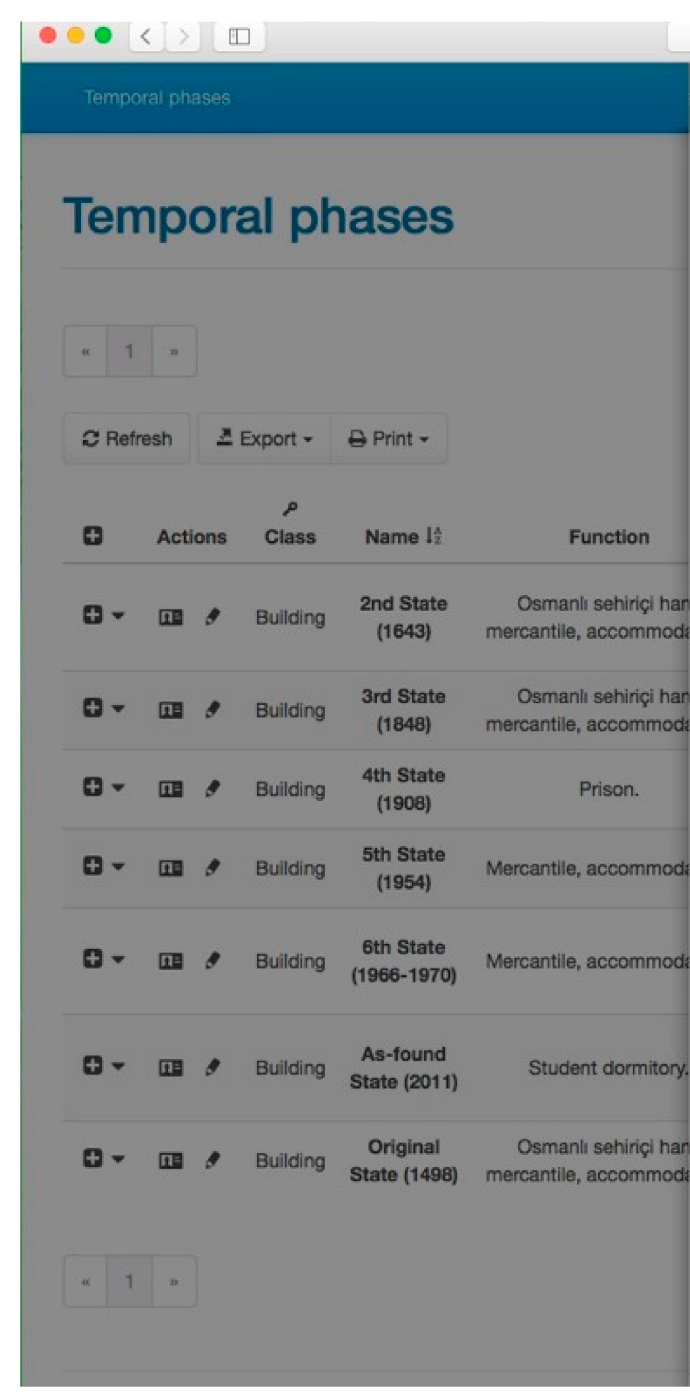

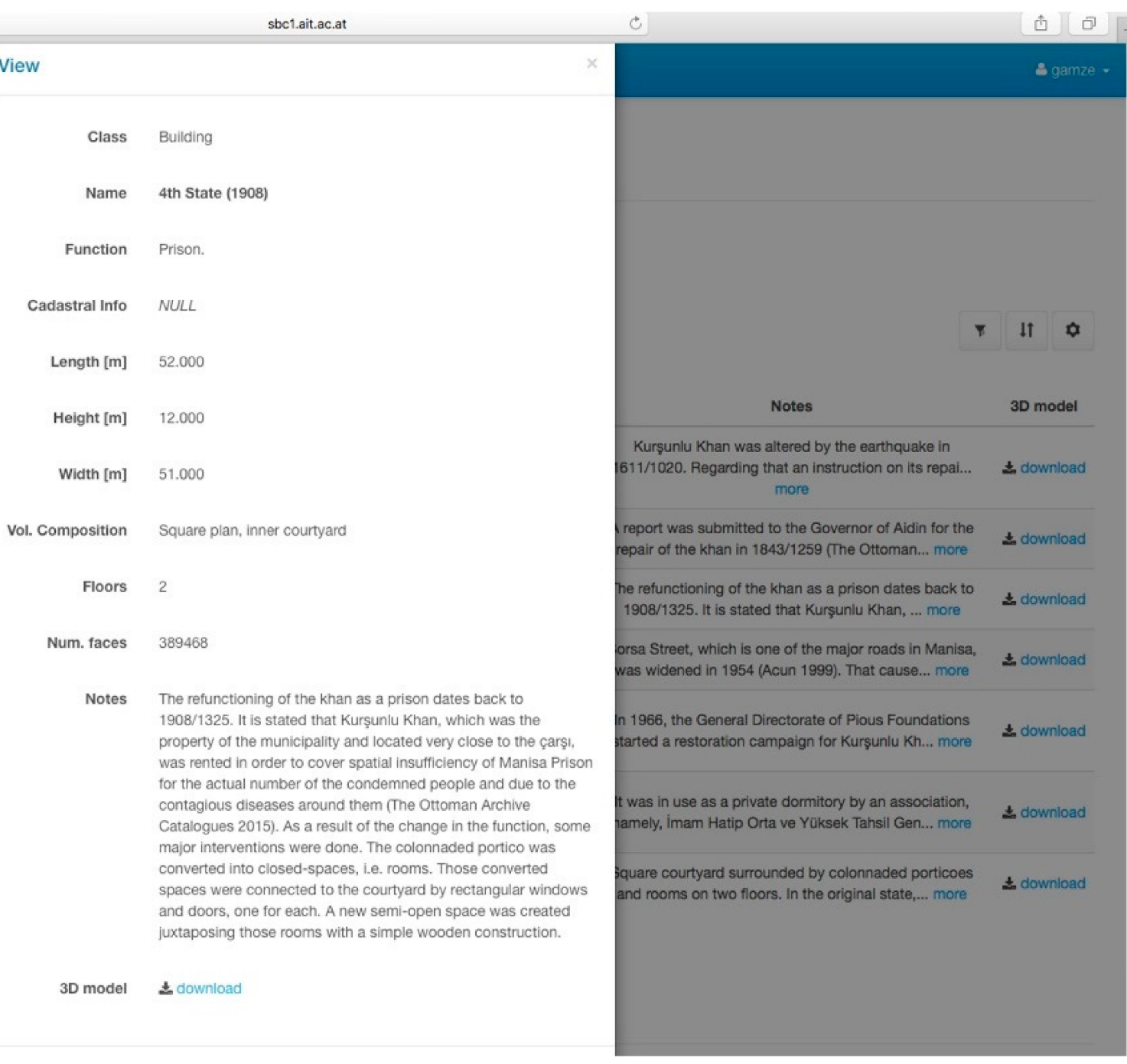

Figure 13. An example of the data visualization in the GUI. 


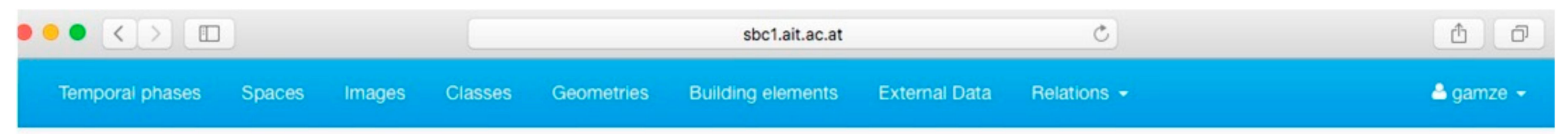

\section{Images per Phase}

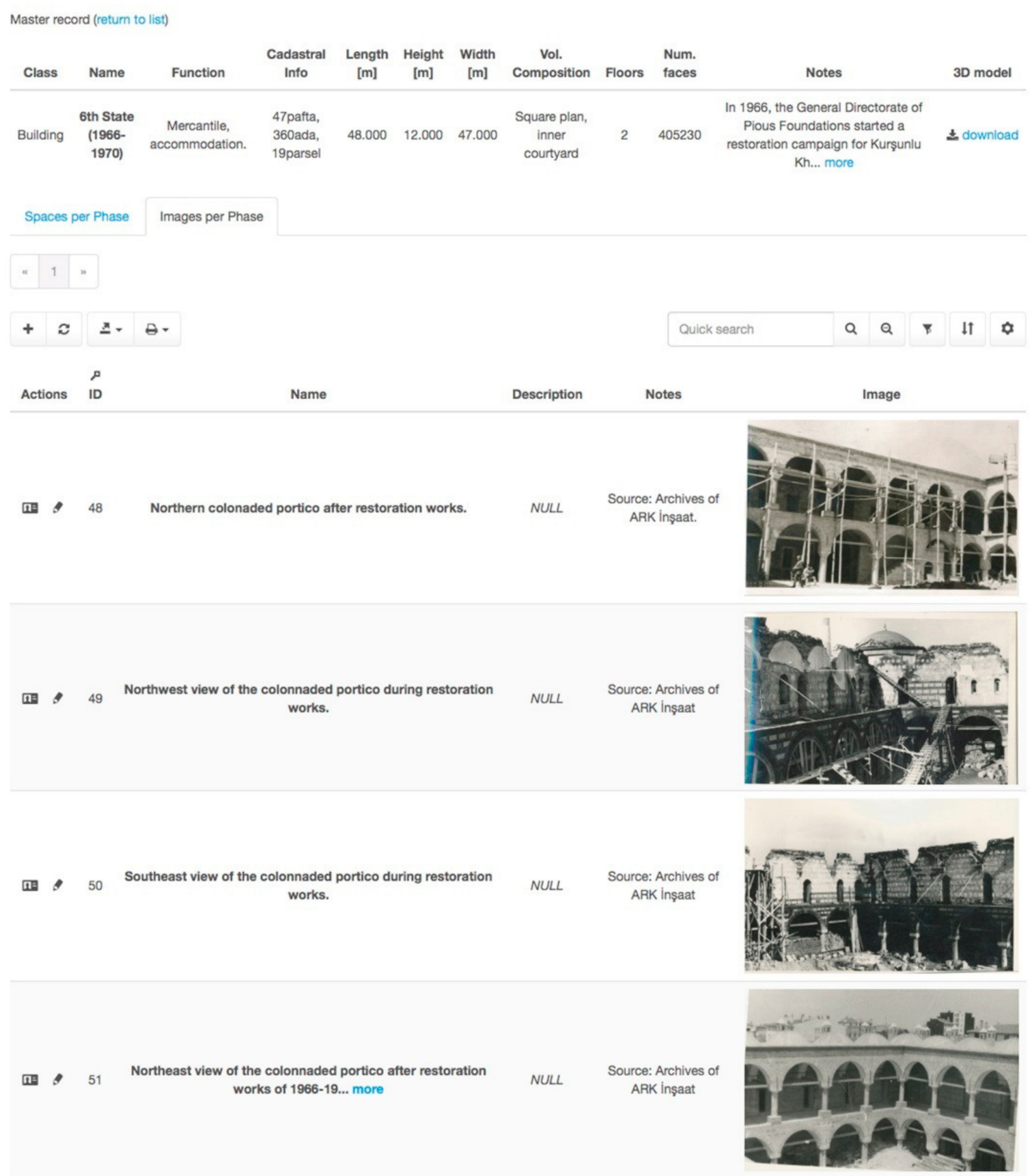

Figure 14. The data visualization in the GUI (images of the 6th state). 


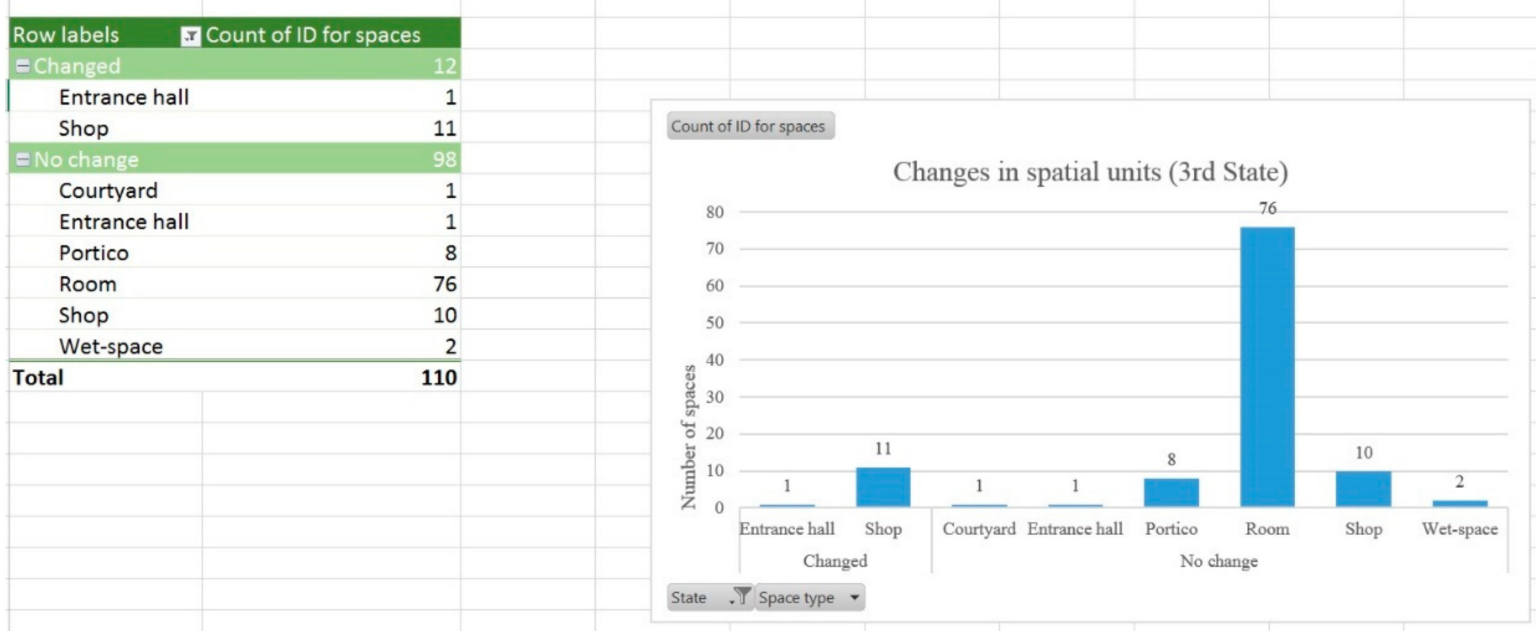

Figure 15. The changes in the spaces making up the 3rd State.

\section{Results}

The prototype of the implemented data repository provides a centralization of the geometric and historical data within a single database, thus, it allows for the complete digital archiving of the building's chronology. This is structured around digital 3D reconstructions of historical phases, allowing access through two different interfaces: the semantically enriched 3D model (for example, via ArcGIS) and a graphical user interface (via web browser), which allows access to information in tabular format. In the former, it is possible to do the inspection of the building's stratigraphy and to apply diverse queries, such as: (i) 3D visualization of different hypothesis historically, (ii) 3D querying of the reliability of these hypothesis, (iii) accessing corresponding written or visual historic sources, (iv) demonstration of historical changes in the construction technique and material culture, (v) learning the different uses/functions with associated sources, and (vi) setting diverse queries for a clearer understanding. Consequently, the visualizations, in terms of the semantically enriched 3D model created in this research, are neither just a volume of spaces nor geometric features. Rather, it is a holistic information container of non-geometric data housed behind the imagery. In contrast to this method, the latter can be reached through a web-based GUI, where the following can be explored: (a) the list of restitution states representing building chronology, their content, and 3D geometric model of each phase, (b) the list of spaces making up each restitution state, (c) the list of building elements making up any space within a restitution state, and (d) the external datasets (for example, historic and legal documents and photographs) related to the building's different restitution states, as primary sources. In addition, those data can be exported, if needed, to software with spreadsheet functionality (for example, Microsoft Excel) for the generation of reports, graphs, or charts.

The proposed prototype described in this article provides systematic archiving and access possibilities for $\mathrm{CH}$ researchers interested in the chronology of a historic building. Through this method, it can support the decision-making process for future interventions pertaining to the qualities and characteristics of the $\mathrm{CH}$ place. Correspondingly, data exploration and retrieval, which is a crucial step for understanding analysis, is made easier in this systemized and holistic environment, and markedly faster due to the integrated data repository. Consequently, the system not only allows for data management purposes, but also allows for the reuse of content and knowledge.

The holistic 3D archive provides a permanent record of $\mathrm{CH}$, prior to any planned or unplanned changes. Data update, revisions, and corrections are possible, which is important as historical buildings are dynamic assets. Instances include a new intervention, repair, or restoration, which can occur on-site. Furthermore, this archive would allow conservation-architects further access to documents or findings in the future, and the ability to add all these changes to the database. 


\section{Discussion}

The integrated management of heterogeneous (geometric, non-geometric, and external data), chronological information of historical buildings is a topic of research where many questions still remain unanswered. The existing approaches are promising and active, but are still limited in perspective. For example, among the existing approaches, there are only a few projects concerning the building chronology of single-scale historical buildings, and despite the use of 3D model being a standard practice, those models are generally limited into raw outline/contour of the $\mathrm{CH}$ entities. Nonetheless, CH researchers need intelligent 3D models which offer a user-friendly environment, along with the fast and efficient retrieval of information for understanding different restitution states throughout history. This requires storage of a huge volume of heterogonous datasets and the capability to manipulate and manage them.

As such, the developed prototype may be an effective tool for the research activities of $\mathrm{CH}$ specialists' by making historical information more visible and readable, which in turn both supports the comprehensive understanding and gives way to a novel (re)thinking. The creation of such a holistic information management approach has crucial importance, as it gives $\mathrm{CH}$ specialists the possibility of using systemized and structured information that provide an entire perception of the building's history. Last but not least, the methodology proposed in this research is a sum of the (re)interpretation of the values and changes specific to the tested archetype, reflecting the notion of Ottoman culture, their way of life, workmanship, construction tradition, and their continuity.

\section{Directions for Future Studies}

The developed solution is a prototype that describes and implements a methodological approach. Hence, the outcomes cover some of the crucial capabilities. However, it should be noted that there is a capacity for further enhancements with architectural conservation-related information. Of foremost importance, the finite details, (for example, cracks, material deteriorations of the as-found state) are not covered by the building elements in the study for the semantically enriched 3D model. Yet, this carries crucial importance in understanding the building and thus, should be enhanced with such information. Another example is how the level of detail could be increased for the analysis of a historic building, covering the structural deformations and their causes, along with the degree and type of material deteriorations. Furthermore, the methodology can be expanded to include any case-specific investigations for conservation, or any other case studies' applications, while the basics of the methodology will remain the same.

The prototype was developed particularly for conservation-architects as the primary actors in architectural heritage conservation, allowing a comprehensive understanding thanks to the holistic repository. Indeed, interpretation by the general public is also important, as public awareness is noted to be of importance in the charters of ICOMOS [28,55]. A future research direction raised here is, thus, to allow new levels of access, especially for the public, in order to develop conservation consciousness and employ e-learning facilities.

Besides, we acknowledge the necessity of a tighter integration of the visualization and querying capabilities without relying on external commercial software, like ArcGIS or Microsoft Excel.

In future applications, spatial modeling, in particular, manual 3D modeling efforts by the adoption of the CAD method, can be redesigned and replaced through the employment of 3D data, based on digital data acquisition. This would require an extensive evaluation of the segmentation process from both the parts and its entirety, a task that would pose a challenge but is of great interest in today's research.

Author Contributions: G.S. evaluated and analyzed the data, G.S. and G.A. designed and implemented the prototype, M.H.-T. supported the evolution of the research, G.S. mainly wrote the paper with contributions by G.A.

Acknowledgments: This paper is an outgrowth of Gamze Saygi's PhD thesis, which dealt with a number of aspects of information management of $\mathrm{CH}$ assets, and has been possible thanks to research fellowship allowing collaboration with Giorgio Agugiaro (first at 3DOM (3D Optical Metrology) Research Unit of FBK, and later at 
Austrian Institute of Technology (AIT)). The authors are thankful to FBK and AIT for providing the IT resources to temporarily host the database and the webserver; Fabio Remondino (FBK) for his constructive criticism, suggestions and support; Ömür Saygın (IYTE) for the useful discussions, and Architect Temel Aktürk on behalf of ARK İnşaat for providing 2D documentation and complementary datasets of the case study.

Conflicts of Interest: The authors declare no conflict of interest. The founding sponsors had no role in the design of the study; in the collection, analyses, or interpretation of data; in the writing of the manuscript, and in the decision to publish the results.

\section{References}

1. Letellier, R. Recording and Information Management for the Conservation of Heritage Places: Guiding Principles. Available online: http:/ / www.getty.edu/conservation/publications_resources/pdf_ publications/recordim.html (accessed on 12 April 2018).

2. El-Hakim, S.; Gonzo, L.; Voltolini, F.; Girardi, S.; Rizzi, A.; Remondino, F.; Whiting, E. Detailed 3D Modelling of Castles. Int. J. Archit. Comput. 2007, 5, 199-220. [CrossRef]

3. Doulamis, A.; Ioannides, M.; Doulamis, N.; Hadjiprocopis, A.; Fritsch, D.; Balet, O.; Santos, P. $4 \mathrm{D}$ reconstruction of the past. In Proceedings of the First International Conference on Remote Sensing and Geoinformation of Environment, Paphos, Cyprus, 5 August 2013.

4. Georgopoulos, A. 3D virtual reconstruction of archaeological monuments. Mediterr. Archaeol. Archaeom. 2014, 14, 155-164.

5. Canciani, M.; Falcolini, C.; Buonfiglio, M.; Pergola, S.; Saccone, M.; Mammí, B.; Van Gool, L. A method for virtual anastylosis: The case of the arch of Titus at the Circus Maximus in Rome. In Proceedings of the IEEE Computer Society Conference on Computer Vision and Pattern Recognition Workshops, Madison, WI, USA, 16-22 June 2003; pp. 2-4.

6. Dudek, I.; Blaise, J.-Y. Understanding Changes in Heritage Architecture. In Proceedings of the IMAGAPP/IVAPP (International Conference on Information Visualization Theroy and Applications), Angers, France, 17-21 May 2010; pp. 91-100.

7. Rodríguez-Gonzálvez, P.; Muñoz-Nieto, A.L.; DelPozo, S.; Sanchez-Aparicio, L.J.; Gonzalez-Aguilera, D.; Micoli, L.; Hejmanowska, B. 4D reconstruction and visualization of cultural heritage: Analyzing our legacy through time. Int. Arch. Photogramm. Remote Sens. Spat. Inf. Sci. 2017, 42, 609-616. [CrossRef]

8. Historic England. Photogrammetric Applications for Cultural Heritage. Guidance for Good Practice; Historic England: Swindon, UK, 2017.

9. AEC (UK) Committee. AEC (UK) BIM Technology Protocol, Practical Implementation of BIM for the UK Architectural, Engineering and Construction (AEC) Industry. Available online: https://aecuk.files. wordpress.com/2015/06/aecukbimtechnologyprotocol-v2-1-1-201506022.pdf (accessed on 12 April 2018).

10. Andrews, D.; Bedford, J.; Bryan, P. Metric Survey Specifications for Cultural Heritage. 2015. Available online: http://archaeologydataservice.ac.uk/archiveDS/archiveDownload?t=arch-1416-1/ dissemination/pdf/9781848021716.pdf (accessed on 12 April 2018).

11. Saygi, G.; Agugiaro, G.; Hamamcıŏlu-Turan, M.; Remondino, F. Evaluation of Gis and Bim Roles for the Information Management of Historical Buildings. ISPRS Ann. Photogramm. Remote Sens. Spat. Inf. Sci. 2013, II-5/W1, 283-288. [CrossRef]

12. Dore, C.; Murphy, M. Current State of the Art Historic Building Information Modelling. Int. Arch. Photogramm. Remote Sens. Spat. Inf. Sci. 2017, XLII-2/W5. [CrossRef]

13. Historic England. BIM for Heritage; Antonopoulou, S., Cons, A., Bryan, P., Eds.; Historic England: Swindon, UK, 2017; Available online: https:/ / content.historicengland.org.uk/images-books/publications/bim-forheritage/heag-154-bim-for-heritage.pdf/ (accessed on 12 April 2018).

14. Hichri, N.; Stefani, C.; De Luca, L.; Veron, P. Review of the "As-Built Bim" Approaches. Int. Arch. Photogramm. Remote Sens. Spat. Inf. Sci. 2013, XL-5/W1, 107-112. [CrossRef]

15. Chiabrando, F.; Lo Turco, M.; Rinaudo, F. Modeling the Decay in an Hbim Starting From 3D Point Clouds. A Followed Approach for Cultural Heritage Knowledge. Int. Arch. Photogramm. Remote Sens. Spat. Inf. Sci. 2017, XLII-2/W5, 605-612. [CrossRef] 
16. Brumana, R.; Oreni, D.; Raimondi, A.; Georgopoulos, A.; Bregianni, A. From survey to HBIM for documentation, dissemination and management of built heritage: The case study of St. Maria in Scaria d'Intelvi. In Proceedings of the 2013 Digital Heritage International Congress (DigitalHeritage), Marseille, France, 28 October-1 November 2013; Volume 1, pp. 497-504.

17. Fai, S.; Filippi, M.; Paliaga, S. Parametric modelling (BIM) for the documentation of vernacular construction methods: a BIM model for the commissariat building, Ottawa, Canada. ISPRS Ann. Photogramm. Remote Sens. Spat. Inf. Sci. 2013, II-5/W1, 115-120. [CrossRef]

18. Yajing, D.; Cong, W. Research on the Building Information Model of the stone building for heritages conservation with the outer south gate of the Ta Keo Temple as an example. In Proceedings of the 2011 International Conference on Electric Technology and Civil Engineering (ICETCE), Lushan, China, 22-24 April 2011; pp. 1488-1491. [CrossRef]

19. Vosselman, G.; Gorte, B.; Sithole, B.G.; Rabbani, B.T. Recognising structure in laser scanner point clouds. Inter. Arch. Photogramm. Remote Sens. Spat. Inf. Sci. 2003, 46, 33-38.

20. $\mathrm{Pu}, \mathrm{S}$; Vosselman, G. Automatic extraction of building features from terrestrial laser scanning. Int. Arch. Photogramm. 2006, 36, 25-27.

21. Rusu, R.B.; Marton, Z.C.; Blodow, N.; Holzbach, A.; Beetz, M. Model-based and learned semantic object labeling in 3D point cloud maps of kitchen environments. In Proceedings of the 2009 IEEE/RSJ International Conference on Intelligent Robots and Systems, St. Louis, MO, USA, 10-15 October 2009; pp. 3601-3608. [CrossRef]

22. Xiong, X.; Huber, D. Using Context to Create Semantic 3D Models of Indoor Environments. In Proceedings of the British Machine Vision Conference, Wales, UK, 31 August-3 September 2010; p. 45. [CrossRef]

23. Yue, K.; Huber, D.; Akinci, B.; Krishnamurti, R. The ASDMCon project: The challenge of detecting defects on construction sites. In Proceedings of the 3D Data Processing, Visualization, and Transmission, Third International Symposium, Chapel Hill, NC, USA, 14-16 June 2006; pp. 1048-1055. [CrossRef]

24. Ben Hmida, H.; Cruz, C. From 3D Point Clouds to Semantic Objects-An Ontology-based Detection Approach. In Proceedings of the International Conference on Knowledge Engineering and Ontology Development, Paris, France, 26-29 October 2011; pp. 255-260.

25. Saygi, G.; Remondino, F. Management of Architectural Heritage Information in BIM and GIS: State-of-the-Art and Future Perspectives. Int. J. Heritage Digit. Era 2013, 2, 695-713. [CrossRef]

26. Centofanti, M.; Continenza, R.; Brusaporci, S.; Trizio, I. the Architectural Information System Siarch3D-Univaq for Analysis and Preservation of Architectural Heritage. Int. Arch. Photogramm. Remote Sens. Spat. Inf. Sci. 2012, 5, 9-14. [CrossRef]

27. SanJosé-Alonso, J.; Finat, J. Information and knowledge systems for integrated models in Cultural Heritage. ISPRS Arch. Photogramm. Remote Sens. Spat. Inf. Sci. 2009, 38. Available online: http:/ / citeseerx.ist.psu.edu/ viewdoc/download?doi=10.1.1.445.2498\&rep=rep1\&type=pdf (accessed on 12 April 2018).

28. ICOMOS. Charter for the Interpretation and Presentation of Cultural Heritage. 2008. Available online: https://www.icomos.org/charters/interpretation_e.pdf (accessed on 12 April 2018).

29. Koller, D.; Frischer, B.; Humphreys, G. Research challenges for digital archives of 3D cultural heritage models. J. Comput. Cult. Heritage 2009, 2, 1-17. [CrossRef]

30. Brusaporci, S. The Importance of Being Honest: Issues of Transparency in Digital Visualization of Architectural Heritage. In Handbook of Research on Emerging Technologies for Architectural and Archaeological Heritage; IGI Global: Hershey, PA, USA, 2017.

31. Apollonio, F.I.; Gaiani, M.; Sun, Z. 3D Modeling and Data Enrichment in Digital Reconstruction of Architectural Heritage. Int. Arch. Photogramm. Remote Sens. Spat. Inf. Sci. 2013, XL-5/W2, 43-48. [CrossRef]

32. Garagnani, S.; Manferdini, A.M. Parametric accuracy: Building information modeling process applied to the cultural heritage preservation. Int. Arch. Photogramm. Remote Sens. Spat. Inf. Sci. 2013, XL-5/W1, 87-92. [CrossRef] 
33. Kolbe, T.H.; Gröger, G.; Plümer, L. CityGML-3D City Models and their Potential for Emergency Response. Available online: https://www.researchgate.net/publication/254899282_Bimserverorg_-_an_Open_Source_ IFC_model_server (accessed on 12 April 2018).

34. Beetz, J.; van Berlo, L.; de Laat, R.; van den Helm, P. bimserver.org-An Open Source IFC Model Server. In Proceedings of the CIB W78 2010: 27th International Conference, Cairo, Egypt, 16-18 November 2010; pp. 16-18. Available online: http:/ / bimserver.org/wp-content/uploads/2010/11/Beetz_Berlo-CIB-W78_ Cairo.pdf (accessed on 12 April 2018).

35. BuildingSMART. 2018. Available online: http://www.buildingsmart-tech.org/ (accessed on 12 April 2018).

36. Ma, Z.; Ren, Y. Integrated Application of BIM and GIS: An Overview. Procedia Eng. 2017, 196, $1072-1079$. [CrossRef]

37. Alp, S.Ç.; Güçhan, N.Ş. Challenges in use of geographical information systems (GIS) in a research for understanding conservation of cultural heritage in Bursa. J. Cult. Heritage Manag. Sustain. Dev. 2017, 7, 328-344. [CrossRef]

38. Baik, A.; Yaagoubi, R.; Boehm, J. Integration of jeddah historical bim and 3D GIS for documentation and restoration of historical monument. Int. Arch. Photogramm. Remote Sens. Spat. Inf. Sci. 2015, XL-5/W7, $29-34$. [CrossRef]

39. Güran, C. Türk Hanlarının Gelişimi ve İstanbul Hanları Mimarisi; Vakıflar Genel Müdürlüğü Yayınları. 1976. Available online: https:/ / books.google.fr/books?id=8tnTnQEACAAJ (accessed on 12 April 2018).

40. UNESCO. Universal Declaration on Cultural Diversity; Stenou, K., Ed.; United Nations Educational, Scientific and Cultural Organization: Paris, France, 2002; Available online: http:/ /scholar.google.com/scholar?hl= en\&btnG=Search\&q=intitle:Universal+Declaration+on+Cultural+Diversity\#3 (accessed on 12 April 2018).

41. Brandi, C.; Basile, G. Theory of Restoration; Istituto Centrale per il Restauro: Roma/Firenze, Italy, 2005.

42. ARK Insaat. Kurşunlu Han Rölöve, Restitüsyon; Restorasyon Projesi; ARK Insaat: Ankara, Turkey, 2011.

43. Agugiaro, G.; Remondino, F.; Girardi, G.; Von Schwerin, J.; Richards-Rissetto, H.; De Amicis, R. QueryArch3D: Querying and Visualising 3D Models of a Maya Archaeological Site in a Web-Based Interface. Geoinform. FCE CTU 2011, 6, 10-17. [CrossRef]

44. Von Schwerin, J.; Richards-Rissetto, H.; Remondino, F.; Agugiaro, G.; Girardi, G. The mayaarch3d project: A 3D webgis for analyzing ancient architecture and landscapes. Lit. Linguist. Comput. 2013, 28, 736-753. [CrossRef]

45. Von Meiss, P. Elements of Architecture: From Form to Place; Routledge: Abingdon-on-Thames, UK, 2011.

46. Booch, G.; Rumbaugh, J.; Jacobson, I. The Unified Modeling Language User Guide. Techniques; 1998; Volume 3. Available online: http:/ / portal.acm.org/citation.cfm?id=1088874 (accessed on 12 April 2018).

47. The PostgreSQL Global Development Group. PostgreSQL 10.3 Documentation. 2018. Available online: https:/ / www.postgresql.org/docs/10/static/index.html (accessed on 12 April 2018).

48. Obe, R.; Hsu, L. PostGIS in Action. Manning. 2011. Available online: https://books.google.fr/books?id= 4kEBRQAACAAJ (accessed on 12 April 2018).

49. Or, S.-H.; Wong, K.; Yu, Y.-K.; Chang, M.; Kong, H.; Highly Automatic Approach to Architectural Floorplan Image Understanding \& Model Generation. Pattern Recognit. 2005, 25-32. Available online: http:/ /www.cse. cuhk.edu.hk/ shor/project/building/vmv05.pdf (accessed on 12 April 2018).

50. Moloo, R.K.; Dawood, M.A.S.; Auleear, A.S. 3-Phase Recognation Approach to Pseudo 3D Building Generation from 2D Floor Plan. Int. J. Comput. Graph. Anim. 2011, 1, 13-27. [CrossRef]

51. Lewis, R.; Sequin, C. Generation of 3D building models from 2D architectural plans. Comput.-Aided Des. 1998, 30, 765-779. [CrossRef]

52. Gimenez, L.; Hippolyte, J.-L.; Robert, S.; Suard, F.; Zreik, K. reconstruction of 3D building information models from 2D scanned plans. J. Build. Eng. 2015, 2, 24-35. [CrossRef]

53. Safe Software Inc. FME ${ }^{\circledR}$ Desktop Training Manual. 2015. Available online: http://docs.safe.com/fme_ training/2015/pdf/FME_Desktop_Training_Manual.pdf (accessed on 12 April 2018). 
54. Cignoni, P.; Cignoni, P.; Callieri, M.; Callieri, M.; Corsini, M.; Corsini, M.; Ranzuglia, G. MeshLab: An Open-Source Mesh Processing Tool. In Proceedings of the Sixth Eurographics Italian Chapter Conference, Salerno, Italy, 2-4 July 2008; pp. 129-136.

55. ICOMOS. Principles for the Recording of Monuments, Groups of Buildings and Sites. 1996. Available online: https:/ / www.icomos.org/charters/archives-e.pdf (accessed on 12 April 2018).

(C) 2018 by the authors. Licensee MDPI, Basel, Switzerland. This article is an open access article distributed under the terms and conditions of the Creative Commons Attribution (CC BY) license (http:/ / creativecommons.org/licenses/by/4.0/). 